\title{
Myeloid C/EBP $\beta$ deficiency reshapes microglial gene expression and is protective in experimental autoimmune encephalomyelitis
}

Marta Pulido-Salgado 1,6, Jose M. Vidal-Taboada ${ }^{1,6}$, Gerardo Garcia Diaz-Barriga ${ }^{2,6}$, Joan Serratosa ${ }^{3}$, Tony Valente ${ }^{1,3,6}$, Paola Castillo ${ }^{4}$, Jonathan Matalonga ${ }^{5}$, Marco Straccia ${ }^{1,2,3,6}$, Josep M. Canals ${ }^{2,6}$, Annabel Valledor $^{5}$, Carme Solà ${ }^{3}$ and Josep Saura ${ }^{1,6^{*}}$

\begin{abstract}
Background: CCAAT/enhancer binding protein $\beta$ (C/EBP $\beta$ ) is a transcription factor that regulates the expression of important pro-inflammatory genes in microglia. Mice deficient for C/EBP $\beta$ show protection against excitotoxic and ischemic CNS damage, but the involvement in this neuroprotective effect of the various C/EBP $\beta$-expressing cell types is not solved. Since $C / E B P \beta$-deficient microglia show attenuated neurotoxicity in culture, we hypothesized that specific C/EBP $\beta$ deficiency in microglia could be neuroprotective in vivo. In this study, we have tested this hypothesis by generating mice with myeloid C/EBP $\beta$ deficiency.

Methods: Mice with myeloid C/EBP $\beta$ deficiency were generated by crossing LysMCre and C/EBP $\beta^{\text {fl/fl }}$ mice. Primary microglial cultures from C/EBP $\beta^{f / / f l}$ and LysMCre-C/EBP $\beta^{f / f l}$ mice were treated with lipopolysaccharide \pm interferon $\gamma$ (IFNy) for $6 \mathrm{~h}$, and gene expression was analyzed by RNA sequencing. Gene expression and C/EBP $\beta$ deletion were analyzed in vivo in microglia isolated from the brains of $C / E B P \beta^{f / / f l}$ and LysMCre-C/EBP $\beta^{f / f l}$ mice treated systemically with lipolysaccharide or vehicle. Mice of LysMCre-C/EBP $\beta^{f / f l}$ or control genotypes were subjected to experimental autoimmune encephalitis and analyzed for clinical signs for 52 days. One- or two-way ANOVA or Kruskal-Wallis with their appropriate post hoc tests were used.
\end{abstract}

Results: LysMCre-C/EBPB ${ }^{f / f l}$ mice showed an efficiency of C/EBP $\beta$ deletion in microglia of 100 and $90 \%$ in vitro and in vivo, respectively. These mice were devoid of female infertility, perinatal mortality and reduced lifespan that are associated to full C/EBP $\beta$ deficiency. Transcriptomic analysis of C/EBP $\beta$-deficient primary microglia revealed C/ EBP $\beta$-dependent expression of 1068 genes, significantly enriched in inflammatory and innate immune responses GO terms. In vivo, microglial expression of the pro-inflammatory genes Cybb, Ptges, II23a, Tnf and Csf3 induced by systemic lipopolysaccharide injection was also blunted by C/EBP $\beta$ deletion. CNS expression of C/EBP $\beta$ was upregulated in experimental autoimmune encephalitis and in multiple sclerosis samples. Finally, LysMCre-C/EBP ${ }^{f / f f l}$ mice showed robust attenuation of clinical signs in experimental autoimmune encephalitis.

Conclusion: This study provides new data that support a central role for C/EBP $\beta$ in the biology of activated microglia, and it offers proof of concept for the therapeutic potential of microglial C/EBP $\beta$ inhibition in multiple sclerosis.

Keywords: Transcription factor, Neuroinflammation, Lipopolysaccharide, Interferon $\gamma$, RNA sequencing

\footnotetext{
* Correspondence: josepsaura@ub.edu

'Department of Biomedicine, Biochemistry and Molecular Biology Unit,

School of Medicine, University of Barcelona, IDIBAPS, Barcelona, Spain

${ }^{6}$ Institute of Neurosciences, University of Barcelona, Barcelona, Spain

Full list of author information is available at the end of the article
} 


\section{Background}

Neuroinflammation is a cellular and molecular response of the CNS to a variety of cues, including pathogens, abnormal protein deposits, toxic metabolites, trauma, autoimmunity or massive cell damage, which has an inflammatory character. Microglia, the innate immune cells of the brain, are the main cell type involved in this response, with astrocytes also playing a prominent role. Chronic and/or exacerbated neuroinflammation is associated with the production of potentially cytotoxic molecules, such as oxygen and nitrogen free radicals, pro-inflammatory cytokines or proteases [1], and this is thought to contribute to neurodegeneration in a growing list of neurological and psychiatric disorders [2].

In neuroinflammation, microglia undergoes massive changes in gene expression that are regulated by a reduced number of transcription factors. There is strong evidence showing that pro-inflammatory gene expression by activated microglia is regulated by the transcription factors NF-kB, AP-1, CREB, STATs, C/EBP $\beta$ and C/EBPS, whereas PPARy and Nrf2 are two of the most important transcription factors regulating anti-inflammatory programs in microglia [3]. Because of their bottleneck position, transcription factors are potential targets to pharmacologically modulate whole cellular programs such as the neuroinflammatory one in activated microglia.

The present study is focused on the role of C/EBP $\beta$, a transcription factor of the b-zip class, in microglial activation. The $\mathrm{C} / \mathrm{EBP} \beta$ gene codes for a single mRNA which can be translated into three protein isoforms, named Full, LAP and LIP, by use of alternative in-frame translation initiation codons [4]. C/EBP $\beta$ levels increase in activated microglia [5] where it regulates the expression of key proinflammatory genes $[3,6-8]$. In vitro and in vivo data suggest a neuroprotective potential for $C / E B P \beta$ inhibition in microglia. Thus, neurotoxicity elicited by activated microglia in neuronal-microglial co-cultures is abolished by the absence of C/EBP $\beta$ in microglia [3]. Also, C/EBP $\beta$ deficient mice show reduced neuronal death and neurological deficits caused by ischemic [9] or excitotoxic [7] damage in vivo. Even though these in vivo findings are promising, ubiquitous $C / E B P \beta$ inhibition is probably undesirable as a therapeutic strategy because C/EBP $\beta$ plays important roles in other cell types, such as adipocytes, hepatocytes or neurons [4], that could be compromised. Our hypothesis is that microglia-targeted C/EBP $\beta$ inhibition could be a safe and effective approach to attenuate neuroinflammation-driven neurodegeneration. In order to test this hypothesis, we generated transgenic mice with myeloid-specific C/EBP $\beta$ deficiency using a Cre-LoxP system and transcriptomically profiled C/EBP $\beta$-deficient microglia. To explore the effects of C/EBP $\beta$ myeloid deficiency in a pathological context, we analyzed the clinical response of these animals to experimental autoimmune encephalomyelitis (EAE), an animal model of multiple sclerosis. The data here presented show that microglial $\mathrm{C} / \mathrm{EBP} \beta$ absence results in remarkable effects on proinflammatory gene expression programs and in the amelioration of EAE symptomatic phenotype.

\section{Methods \\ Human samples}

Postmortem human temporal cortex samples were supplied by the Neurological Tissue Bank of the BiobancHospital Clínic-IDIBAPS (Barcelona, Spain): healthy controls $(n=4 ; 2$ ㅇ, 2 ; ; age, $66-81$ years; postmortem delay, 3.5-23.5 h) and patients with a diagnosis of primary progressive MS $(n=5 ; 2$ ㅇ, 3 ; age, $46-68$ years; postmortem delay, $3-8 \mathrm{~h}$ ). Protein was extracted from frozen tissue blocks [10], and it was processed for Western blot analysis as described below.

\section{Animals}

Mice with myeloid C/EBP $\beta$ deficiency were generated by crossing transgenic mice expressing Cre-recombinase under the lysozyme M (LysM) promoter (B6.129P2Lyz $^{\operatorname{tm} 1(\text { (cre)Ifo } / J ~ 004781, ~ J a c k s o n ~ L a b o r a t o r i e s) ~ w i t h ~ m i c e ~}$ carrying a C/EBP $\beta$ gene flanked by LoxP sites [11] kindly donated by Prof Esta Sterneck (National Cancer Institute, Frederick, MD). These mice are referred to as LysMCre-C/EBP $\beta^{\mathrm{fl} / \mathrm{fl}}$ in the manuscript. LysMCre, C/ $E B P \beta^{\mathrm{fl} / \mathrm{fl}}$ and LysMCre-C/EBP $\beta^{\mathrm{f} / \mathrm{ll}}$ colonies, all on a C57BL/ 6 background, were intercrossed every three generations to diminish inbreeding. Mouse tail samples were used for genotyping the presence of the Cre transgene and the floxed C/EBP $\beta$ alleles. DNA was extracted and purified using KAPA Mouse Genotyping Kit (Kapa Biosystems). One microliter of the supernatant was used for polymerase chain reaction (PCR) amplification with specific primers for the LysMCre (5'-CCCAGAAATGCCAGATTACG-3' mutant, 5' -CTTGGGCTGCCAGAATTTCTC-3' common, 5' -TTACAGTCGGCCAGGCTGAC-3' wild type) as described in the JAX mouse database (The Jackson Laboratory) and the C/EBP $\beta$ floxed allele (forward: 5GAGCCACCGCGTCCTCCAGC-'3', reverse: 5'-GGT CGGTGCGCGTCATTGCC-3'). PCR products were loaded on $2 \%$ agarose gels to check the presence (700 bp) or absence (350 bp) of the LysMCre transgene and the wild-type (240 bp) or floxed (320 bp) C/EBP $\beta$ allele. The mice were breed and housed under specific pathogen-free conditions in the animal facilities at the School of Medicine, University of Barcelona.

\section{Primary microglial cultures}

Microglial cells were isolated from primary mixed glial cultures prepared from P1-P3 C/EBP $\beta^{\mathrm{fl} / \mathrm{fl}}$ and LysMCre$\mathrm{C} / \mathrm{EBP} \beta^{\mathrm{fl} / \mathrm{fl}}$ mice. The brains were dissected, the meninges removed and the cortices digested with $0.25 \%$ trypsin for 
$30 \mathrm{~min}$ at $37^{\circ} \mathrm{C}$. Trypsinization was stopped by adding an equal volume of culture medium (Dulbecco's modified Eagle's medium-F-12 nutrient mixture, fetal bovine serum 10\%, penicillin $100 \mathrm{U} / \mathrm{mL}$, streptomycin $100 \mu \mathrm{g} / \mathrm{mL}$ and amphotericin B $0.5 \mu \mathrm{g} / \mathrm{mL}$ ) with $160 \mu \mathrm{g} / \mathrm{mL}$ deoxyribonuclease I (all from Invitrogen or Sigma) and brought to a single cell suspension by repeated pipetting followed by passage through a $100-\mu \mathrm{m}$ pore mesh. The solution was pelleted (7 $\mathrm{min}, 200 \mathrm{~g}$ ) and resuspended in culture medium. Glial cells were seeded at a density of $3.5 \times 10^{5}$ cells $/ \mathrm{mL}$ and cultured at $37{ }^{\circ} \mathrm{C}$ in humidified $5 \% \mathrm{CO}_{2}-$ 95\% air. Medium was replaced once a week. Microglial cultures were prepared from DIV19-21 mixed glial cultures by the mild trypsinization method [12] and used $24 \mathrm{~h}$ after isolation.

\section{Ex vivo isolation of adult microglia}

Microglial cells were acutely isolated from male adult mouse brains as described [13]. Briefly, mice were deeply anesthetized with isofluorane, perfused transcardially with ice-cold $\mathrm{PBS}+10 \mathrm{U} / \mathrm{ml}$ heparin and the brains dissected and dissociated by trypsin digestion as described in the "Primary microglial cultures" section. Dissociated cells were then resuspended in 30\% Percoll (GE Healthcare) and centrifuged for $10 \mathrm{~min}$ at $700 \mathrm{~g}$. The myelin-containing supernatant was removed, and the pelleted cells were washed twice with ice-cold PBS. Cells were then incubated with $10 \mu \mathrm{l}$ CD11b Microbeads (Miltenyi Biotec) and $90 \mu \mathrm{l}$ buffer (PBS supplemented with 0.5\% BSA and $2 \mathrm{mM}$ EDTA) for $15 \mathrm{~min}$ at $4{ }^{\circ} \mathrm{C}$. After washing, CD11b+ cells were separated in a magnetic field using MS columns (Miltenyi Biotec). The CD11b+ fraction was collected and used for further analyses. The yield was 379.415 \pm 79.073 (SD, $n=48$ ) microglial cells per brain.

\section{In vivo systemic lipopolysaccharide (LPS) injection}

Systemic LPS injection was used as an in vivo model of acute neuroinflammatory response. Eight-week-old male mice were injected i.p. with $4 \mathrm{mg} / \mathrm{kg}$ LPS (055:B5, Sigma-Aldrich; $100 \mu \mathrm{l}$ per animal) or vehicle (PBS), and microglia was isolated $16 \mathrm{~h}$ after LPS injection as described above.

\section{Experimental autoimmune encephalomyelitis}

EAE, an animal model of multiple sclerosis, was used to analyze the effect of microglial $C / E B P \beta$ deficiency in an in vivo model of a neurological disorder. LysMCre, $\mathrm{C} /$ $\mathrm{EBP} \beta^{\mathrm{fl} / \mathrm{fl}}$ and LysMCre-C/EBP $\beta^{\mathrm{fl} / \mathrm{fl}}$ 7-8-week-old female mice were used. Under isofluorane anesthesia, the animals were subcutaneously injected at two sites into the flanks with $200 \mu \mathrm{l}$ of a freshly prepared immunization cocktail containing myelin oligodendrocyte glycoprotein peptide (MOG3555; Sigma; $100 \mu \mathrm{g} /$ mouse), complete Freund's adjuvant (Sigma) and Mycobacterium tuberculosis (H37R; Difco;
$1 \mathrm{mg} /$ mouse). Control mice received immunization cocktail without MOG35-55. Immediately after immunization and 2 days later, mice were injected i.p. with Bordetella pertussis toxin (Sigma, $500 \mathrm{ng} / \mathrm{mouse}$ ). Mice were weighed and scored daily from day 8 post-immunization in a blind manner according to the following scale: 0 , no deficit; 1 , tail paralysis; 2, hind limb paresis; 3 , incomplete hind limb paralysis; 4, complete hind limb paralysis; and 5, moribund state or death. Wet food was supplied when score 2 was reached and $200 \mu \mathrm{L}$ of saline was administrated subcutaneously when animals scored 4. For comparison of EAE progression among genotypes, mice were scored until 52 days post-immunization at which point they were sacrificed. For analysis of $\mathrm{C} / \mathrm{EBP} \beta$ messenger RNA (mRNA) and protein expression in EAE, CFA- and MOG35-55-treated wildtype mice were sacrificed at various time-points postimmunization (9, 14, 21 and 28 days). The spinal cords were dissected into cervical, thoracic and lumbar regions, whereas the brains were dissected into hindbrain, midbrain and forebrain. Tissue samples were quickly frozen and stored at $-80{ }^{\circ} \mathrm{C}$.

\section{Immunocytochemistry}

Cultured cells were fixed with $4 \%$ paraformaldehyde in PBS for $20 \mathrm{~min}$ at RT. After permeation with chilled methanol for $7 \mathrm{~min}$ and three PBS rinses, cells were incubated overnight at $4{ }^{\circ} \mathrm{C}$ with the primary antibody diluted in $7 \%$ normal goat serum (Sigma) in PBS containing $0.01 \%$ sodium azide. After rinsing in PBS, cells were incubated for $1 \mathrm{~h}$ at RT with secondary antibody and DAPI $(5 \mu \mathrm{g} / \mathrm{mL})$. The primary antibodies were monoclonal mouse anti-C/EBP $\beta$ (1:500, Abcam, ab-18336), polyclonal rabbit anti-nitric oxide synthase 2 (NOS2) (1:400, BD Transduction Laboratories, 610333), polyclonal rabbit anti-GFAP (1:1000, DakoCytomation, Z0334), polyclonal rabbit anti-Iba1 (1:1000, Wako, 019-19741) and monoclonal rat anti-CD11b (1:300, Serotec, MCA711G, clone 5C6). The secondary antibodies were goat antimouse Alexa 546 (1:1000, Molecular Probes, A-11018), Alexa 488 (1:1000, Molecular Probes, A11017), goat antirabbit, Alexa 488 (1:1000, Molecular Probes, A-11070) and goat anti-rat Alexa 546 (1:1000, Molecular Probes, A11081). Microscopy images were obtained with an Olympus IX70 microscope and a digital camera (CC-12, Soft Imaging System GmbH).

\section{Cytospin}

25.000 microglial cells from one adult mouse brain isolated as described in the "Ex vivo isolation of adult microglia" section were centrifuged for $5 \mathrm{~min}$ at $1000 \mathrm{rpm}$ at RT using Shandon Cytospin 4 (Thermo Scientific) and collected in gelatinized slides. Cells were then fixed with $4 \%$ paraformaldehyde in PBS for $20 \mathrm{~min}$ at RT and washed three times in PBS. After blocking for $30 \mathrm{~min}$ at RT with PBS containing 
$1 \%$ BSA, $0.03 \%$ Triton and $10 \%$ normal donkey serum (Gibco), cells were incubated overnight at $4{ }^{\circ} \mathrm{C}$ with primary antibodies diluted in blocking solution. After washing in PBS, slides were incubated for $1 \mathrm{~h}$ at RT with secondary antibodies and DAPI $(5 \mu \mathrm{g} / \mathrm{mL})$ diluted in blocking solution. The primary antibodies were monoclonal mouse anti-C/EBP $\beta$ (1:1111, AbCam, ab-18336) and monoclonal rat anti-CD68 (1:1000, Serotec, MCA1957). The secondary antibodies were donkey anti-mouse Alexa 546 (1:1000, Molecular Probes, A21203) and goat anti-rat Alexa 488 (1:1000, Molecular Probes, A21208). Microscopy images were taken with a Nikon Eclipse E 1000 microscope and a digital camera Olympus DP72.

\section{Histology}

$\mathrm{C} / \mathrm{EBP} \beta^{\mathrm{f} / \mathrm{fl}}$ and LysMCre-C/EBP $\beta^{\mathrm{f} / \mathrm{fl}} 10$-week-old female mice were deeply anesthetized with isofluorane and perfused transcardially with ice-cold $4 \%$ paraformaldehyde in PBS. The brain, lung, heart, mammary gland, spleen, liver, lung, kidney, femur bone and lumbar vertebrae were carefully dissected. Initial examination of macroscopic appearance of the organs was performed by reporting any gross anormality. Tissue specimens were formalin-fixed, paraffin-embedded using routine procedures and then cut into $5-\mu \mathrm{m}$ semi-serial sections. Every fifth slide was stained with haematoxylin and eosin and examined under light microscope.

\section{NO determination}

Primary microglial cultures from $\mathrm{C} / \mathrm{EBP} \beta^{\mathrm{f} / \mathrm{fl}}$ and LysMCre$\mathrm{C} / \mathrm{EBP} \beta^{\mathrm{f} / \mathrm{fl}}$ mice were treated with LPS $(100 \mathrm{ng} / \mathrm{mL})$ with or without IFNY $(0.1,1,10$ or $30 \mathrm{ng} / \mathrm{mL})$ for $48 \mathrm{~h}$. NO production was assessed by detecting accumulation of nitrites in the conditioned medium by the Griess colorimetric assay, as described [12].

\section{Isolation of total proteins and Western blot}

Total microglial proteins were isolated by lysing pelleted cells from one $75 \mathrm{~cm}^{2}$ flask of primary microglial cultures per treatment condition (LPS $100 \mathrm{ng} / \mathrm{mL}$ with or without IFNy $1 \mathrm{ng} / \mathrm{mL}$ for $24 \mathrm{~h}$ ) and CD11b+ cells isolated from one adult brain as described in the "Ex vivo isolation of adult microglia" section with $50 \mu \mathrm{L}$ of RIPA buffer (containing Igepal CA-630 $(10 \mu \mathrm{L} / \mathrm{mL})$, sodium deoxycholate $(5 \mathrm{mg} / \mathrm{mL})$, SDS $(1 \mathrm{mg} / \mathrm{mL})$ and protease inhibitor cocktail Complete, in PBS. Protein amount was determined by the Bradford assay, and protein samples $(20 \mu \mathrm{g})$ were denatured $\left(5 \mathrm{~min} 95^{\circ} \mathrm{C}\right)$, resolved by SDS-PAGE on $12 \%$ gels and transferred to a PDVF membrane. Membranes were incubated overnight at $4{ }^{\circ} \mathrm{C}$ with primary anti-C/EBP $\beta$ antibodies of mouse origin (Abcam, ab18336; for primary cultured microglia proteins) or rabbit origin (Abcam, ab32358; for acutely isolated adult microglia proteins) diluted in both cases 1:500 in immunoblot buffer (Tris-buffered saline (TBS) containing $0.05 \%$ Tween-20 and 5\% nonfat dry milk). Following three TBS-0.05\% Tween-20 rinses, HRP-labelled anti-mouse (1:2000, Santa Cruz, sc-2055) and anti-rabbit (1:15000, GE Healthcare, NA934) secondary antibodies were incubated for $45 \mathrm{~min}$ at RT. To label the loading control protein, monoclonal mouse anti- $\beta$-actin-peroxidase $(1 / 40000$, Sigma, A3854) was incubated for $20 \mathrm{~min}$ at RT. Proteins were visualized with WesternBright ${ }^{\mathrm{tw}}$ Quantum-ECL (Advansta) and membranes exposed to Agfa Curix RP2 Plus films. Data are expressed as the ratio between the intensity of the $C / E B P \beta$ band and the loading control protein band ( $\beta$-actin). For Western blot with EAE and human postmortem samples, the same protocol was used with the exception that the primary antibody was co-incubated with an enhancer solution (Signal Boost ${ }^{\mathrm{Tm}}$ Immunoreaction Enhancer Kit, Merck).

\section{Total RNA extraction and qRT-PCR}

Isolation of total RNA from microglial cell cultures was performed by lysing pelleted cells from one $75 \mathrm{~cm}^{2}$ flask per treatment condition (LPS $100 \mathrm{ng} / \mathrm{mL}$ with or without IFN $\gamma 1 \mathrm{ng} / \mathrm{mL}$ for $6 \mathrm{~h}$ ) with $1 \mathrm{~mL}$ of TriReagent (Sigma) and $100 \mu \mathrm{l}$ of 1-bromo-3-chloropropane (BCP, Sigma). The aqueous phase containing total RNA was recovered after centrifugation for $15 \mathrm{~min}$ at $12.000 \mathrm{~g}$ at $4{ }^{\circ} \mathrm{C}$, mixed with an equal volume of ice-cold $70 \%$ ethanol and loaded onto a PureLink $k^{\text {in }}$ Micro Kit column (Invitrogen). Total RNA was then purified following manufacturer's instructions. Isolation of total RNA from adult microglial cells was carried on after pelleting CD11b+ cells isolated from one adult brain as described in the "Ex vivo isolation of adult microglia" section, using PureLink $^{\text {tm }}$ Micro Kit (Invitrogen) following manufacturer's instructions. Isolation of total RNA from spinal cord (cervical, thoracic and lumbar) and brain (mesencephalon plus diencephalon, rhombencephalon and telencephalon) samples of EAE mice was performed using Trizol method (Tri ${ }^{\circ}$ Reagent, Sigma-Aldrich). Total RNA was quantified spectrophotometrically with Nano Drop ND-1000 (Thermo Scientific). Reverse transcription reactions were carried out from $300 \mathrm{ng}$ (cultured cells) or $1 \mu \mathrm{g}$ (tissue) of total RNA with random primers using Transcriptor Reverse Transcriptase (Roche). Complementary DNA (cDNA) was diluted $1 / 30$, and $3 \mu$ l were used to perform qRT-PCR with qPCRBIO Sygreen Mix Lo-ROX (PCB-P20.11-50, Vitro) in $15 \mu \mathrm{l}$ of final volume reaction using CFX96 Thermal Cycler equipment (Bio-Rad). Measurements were performed in duplicates. Primers, shown in Table 1, were used at final concentration of $300 \mathrm{nM}$. Samples were run for 45 cycles $\left(95^{\circ} \mathrm{C}\right.$ for $30 \mathrm{~s}, 60{ }^{\circ} \mathrm{C}$ or $62{ }^{\circ} \mathrm{C}$ for $1 \mathrm{~min}$ and $72{ }^{\circ} \mathrm{C}$ for $30 \mathrm{~s}$ ). Amplification specificity was confirmed by the analysis of melting curves, and relative gene expression values were calculated using Bio- 
Table 1 Primers used in this study

\begin{tabular}{lll}
\hline Gene & Forward & Reverse \\
\hline Cebpb & AAG CTG AGC GAC GAG TAC & TCA GCT CCA GCA CCT TGT G \\
& AAG A & \\
I23a & TGT GCC CCG TAT CCA GTG TG & AAA AGC CAG ACC TTG GCG GA \\
Cybb & ACT CCT TGG GTC AGC ACT GGC & GCA ACA CGC ACT GGA ACC CCT \\
Tnfa & TGA TCC GCG ACG TGG AA & ACC GCC TGG AGT TCT GGA A \\
Ptges & AGG CCA GAT GAG GCT GCG GA & AGC GAA GGC GTG GGT TCA GC \\
Csf3 & AGA GCT GCA GCC CAG ATC ACC & AGC TGC AGG GCC ATT AGC \\
& TTC A \\
Rn18s & GTA ACC CGT TGA ACC CCA TT & CCA TCC AAT CGG TAG TAG CG \\
Hprt1 & ATC ATT ATG CCG AGG ATT TGG & GCA AAG AAC TTA TAG CCC CC \\
Sdha & TGG GGA GTG CCG TGG TGT CA & CAT GGC TGT GCC GTC CCC TG
\end{tabular}

Rad CFX Managing software (Bio-Rad) with the comparative Ct or $\Delta \Delta \mathrm{Ct}$ method.

\section{Viability}

Microglial cell cultures from $\mathrm{C} / \mathrm{EBP} \beta^{\mathrm{fl} / \mathrm{fl}}$ and LysMCre$\mathrm{C} / \mathrm{EBP} \beta^{\mathrm{f} / \mathrm{fl}}$ mice were treated with LPS $1 \mu \mathrm{g} / \mathrm{mL}$ with or without IFNY 1 or $30 \mathrm{ng} / \mathrm{mL}$. Cells were fixed $(0,3,5$ or 7 days post-treatment) and probed as described in the "Immunocytochemistry" section. DAPI and Iba1-positive cells were manually counted using Image s software. In every experiment $(n=3)$, three wells per condition and three fields per well were analyzed. Results are represented as percentage of live cells on day 0 .

\section{Phagocytosis}

The Salmonella enterica serovar Typhimurium (S. typhimurium) SV5015 strain, a His+ derivative of the SL1344 strain (mouse-virulent) [14], was transformed with the pBR.RFP.1 plasmid [15] to render red autofluorescent bacterial cells. To test microglia phagocytic capacity, first, microglial cell cultures from $\mathrm{C} / \mathrm{EBP} \beta^{\mathrm{fl} / \mathrm{fl}}$ and LysMCre-C/ $\mathrm{EBP} \beta^{\mathrm{fl} / \mathrm{fl}}$ mice were treated with LPS $100 \mathrm{ng} / \mathrm{mL}$ with or without IFNY $1 \mathrm{ng} / \mathrm{mL}$ in antibiotic-free culture medium. Twenty-four hours after treatment, microglia were infected with S. typhimurium for 30 min at multiplicity of infection of 5 , defined as the ratio of bacteria per cultured cell. Non-ingested bacilli were eliminated by washing three times with PBS. Cells to assess phagocytic activity were then fixed as described in the "Immunocytochemistry" section, whereas to study resolution of infection, microglial cells were further incubated for $1 \mathrm{~h}$ in medium containing $100 \mu \mathrm{M}$ gentamicin (Sigma-Aldrich) to kill extracellular bacteria and then switched to medium with a lower dose of gentamicin $(10 \mu \mathrm{M})$ for $3 \mathrm{~h}$ before fixation. Cells were immunostained for Iba1 as described in the "Immunocytochemistry" section. Intracellular autofluorescent bacteria were manually counted using the ImageJ software along with DAPI and Iba1-positive cells. In every experiment $(n=3)$, two wells for each condition and three fields per well were analyzed. Results are represented as percentage of infected microglial cells and number of bacteria phagocytosed at 0.5 and $4 \mathrm{~h}$ post Salmonella infection.

\section{RNA sequencing (RNAseq)}

Total RNA from primary microglia was isolated as described in the "Total RNA extraction and qRT-PCR" section. Total RNA integrity and quality were assessed with the Bioanalyzer 2100 system (Agilent). Library preparation and ultrasequencing were performed following Illumina's (San Diego, CA) protocols. First, transfer RNA (tRNA) and ribosomal RNA (rRNA) were removed from $1 \mu \mathrm{g}$ of total RNA using TruSeq Stranded Total RNA Sample Prep Kits (Illumina). Then, the RNA pool (mRNA + miRNA + IncRNA + other RNAs) was fragmented into pieces of approximately $200 \mathrm{bp}$ using divalent cations under elevated temperature. The cleaved RNA fragments were reversetranscribed into first-strand cDNA using reverse transcriptase and random primers. Next, the second strand was synthesized using DNA polymerase I and RNAse $\mathrm{H}$. These double-stranded cDNA fragments were endrepaired by T4 DNA polymerase and Klenow DNA polymerase, and phosphorylated by $\mathrm{T} 4$ polynucleotide kinase. The cDNA products were incubated with Klenow DNA polymerase to generate 39 adenine overhangs, therefore allowing ligation to Illumina indexing adapters to the double-stranded cDNA ends. The adapter-ligated products were purified with Ampure XP magnetic beads (Agencourt Bioscience Corporation, Beverly, MA, USA), and libraries were amplified by 15 cycles of PCR with Phusion DNA polymerase (Finnzymes Reagents, Vantaa, Finland). Constructed libraries were validated and quantified using BioRad's automated electrophoresis system Experion and qRT-PCR, respectively. Pools of six indexed libraries were mixed (multiplexed) at equimolar ratios to yield a total oligonucleotide mix concentration of $10 \mathrm{nM}$. Finally, the resulting libraries were sequenced on the Genome Analyzer IIx platform (Illumina) to generate $150 \mathrm{bp}$ single reads. Six pooled indexed libraries were sequenced in each flow cell lane. Raw sequence (FASTQ format) were processed through a series of sequential steps: (1) aggressive adapters removal; (2) alignment/mapping of RNA sequences to the mouse genome reference (Mus musculus.mm10) using tophat software; and (3) sorting and cataloguing of the results by using Samtools software [16] in Bam files.

\section{RNAseq statistic and bioinformatic analyses}

Bam files of RNA readings were processed using the Rsubread package [17] in the R environment and aligned to the mm10 version of mouse genome with the function featureCounts [18]. Summarized readings by gene were then normalized using voom normalization [19] to fit the count matrix into linear modelling with the 
package limma [20]. Normalized whole read counts were used to cluster samples with package hcluster using standard hierarchical cluster with average linkage. A linear fit model algorithm was used to obtain differentially expressed genes (DEGs) that complied with $p<0.01$ and fold change $>2$. DEGs by comparison were summarized using the VennDiagram package. Heatmap visualization and clustering of DEGs was done with Genesis software after expressing gene values in standard deviation and using hierarchical clustering with average linkage by genes and samples. Finally, Genecodis [21] tools were used to obtain Gene Ontology and Kyoto Encyclopedia of Genes and Genomes (KEGG) pathway terms enrichment using the adjusted $p$ value $<0.01$ after a hypergeometric test. Reads per kilobase per million mapped reads (RPKM) filtered values (sum of RPKM $>2$ for each gene) were introduced to the Weighted Correlation Gene Network Analysis (WGCNA) package in R, to perform WGCNA as previously described [22]. Combined samples were surveyed and measured with the pickSoftThreshold function to obtain a correct power $\beta$ considering the smallest value to give a free-scale topology. Using 9 as the power value, the blockwise function was used with parameters mergeCutHeight $=0.001$ and detectCutHeight $=0.995$ to obtain 34 modules codenamed by color, containing genes with high coexpression similarity. Module relationship with traits: treatment $($ Vehicle $=0 ; \mathrm{LPS}=1 ; \mathrm{LPS}+\mathrm{IFN} \gamma=2)$ and genotype $\left(\mathrm{C} / \mathrm{EBP}^{\mathrm{fl} / \mathrm{fl}}=0\right.$; LysMCre-C/EBP $\left.\beta^{\mathrm{fl} / \mathrm{fl}}=1\right)$ was also calculated for each module to obtain a correlation. Gene symbols and normalized values were appended, and heatmap visualization of individual modules was obtained within the WGCNA package in R. Finally the Metacore ${ }^{\mathrm{Tx}}$ platform was used to obtain the described interactions of proteins among the different modules to produce "external knowledge networks", while VisAnt software was used with the correlation values in our experiments to produce the specific network of correlation within our data. For the separate analysis of DEGs in each experimental condition, samples of each group (control, LPS, LPS + IFN $\gamma$ ) were used in a separate pipeline, then a linear fit algorithm with Bayesian correction was applied grouping biological replicates of samples and designing a contrast matrix that compared genotypes selecting genes which complied with criteria fold change $>2$, adjusted $p<0.05$ (Benjamini-Hochberg procedure) as sample number was lower and power diminished for these analyses. DEGs in these lists were mostly in agreement with the whole comparison matrix DEGs.

\section{Statistics}

Statistical analyses of RNAseq data are described in the "RNA sequencing (RNAseq)" section. For other parts of the study, statistical analyses were performed using one- way ANOVA followed by Newman-Keuls post hoc test when three or more experimental groups were compared. When the effect of treatment on genotype or the opposite were studied, two-way ANOVA followed by Bonferroni post-test was used. Non-parametric measures (EAE scores) were analyzed with Kruskal-Wallis test and Dunn's multiple comparison post-test. Values of $p<0.05$ were considered statistically significant. Results are represented as mean \pm standard error of the mean (SEM). Experimental data were analyzed using GraphPad Prism 5.01 software.

\section{Results}

\section{Characterization of LysMCre-C/EBP $\beta^{\mathrm{fl} / \mathrm{fl}}$ mice and their microglia in primary culture}

LysMCre-C/EBP $\beta^{\mathrm{fl} / \mathrm{fl}}$ mice had normal appearance and body weight (Fig. 1a) and showed no obvious behavioral abnormalities when compared to wild type, LysMCre or $\mathrm{C} / \mathrm{EBP}^{\mathrm{fl} / \mathrm{fl}}$ mice. Their survival rate and fertility were normal in contrast to what has been reported in C/EBP $\beta$ knockout mice [23]. Litter size and gender distribution were also normal. Histological evaluation of haematoxylin and eosin stained sections (Fig. 1b) showed normal mammary gland and red and white spleen pulp morphology, again in contrast to reports in mice with ubiquitous $\mathrm{C} /$ EBP $\beta$ deficiency [23]. No differences between genotypes were observed in all other tissues and organs examined, listed in the online methods section.

Western blot analysis of proteins from primary microglial cultures showed robust $C / E B P \beta$ expression in $C / E B P \beta^{\mathrm{f} / \mathrm{fl}}$ microglia, and this expression was enhanced by LPS + IFNy treatment (Fig. 2a). Interestingly, C/EBP $\beta$ proteins were not detected in primary microglia from LysMCre-C/EBP $\beta^{\mathrm{f} / \mathrm{fl}}$ mice, neither in control nor after LPS + IFN $\gamma$ treatment (Fig. 2a, b). Immunocytochemical C/EBP $\beta$ staining confirmed this finding. In control conditions, $C / E B P \beta$ was present in most microglial cells in primary cultures from $\mathrm{C} / \mathrm{EBP} \mathrm{\beta}^{\mathrm{f} / \mathrm{fl}}$ mice, and the number of positive cells and the intensity of the staining was enhanced by LPS + IFNy treatment $(24 \mathrm{~h})$. In contrast, microglia from LysMCre-C/ $\mathrm{EBP} \beta^{\mathrm{f} / \mathrm{fl}}$ mice were devoid of C/EBP $\beta$ immunostaining, both in control and LPS + IFN $\gamma$ conditions (Fig. 2c). These findings show that the efficiency of LysMCreinduced recombination of C/EBP $\beta$ LoxP sites in primary microglia from LysMCre-C/EBP $\beta^{\mathrm{fl} / \mathrm{fl}}$ mice is near $100 \%$. C/EBP $\beta$ immunocytochemistry of mixed glial cultures confirmed the absence of C/EBP $\beta$ in LysMCre-C/EBP $\beta^{\mathrm{fl} / \mathrm{fl}}$ microglia, identified by Iba1 immunostaining (Fig. 2e), and showed the presence of normal levels of C/EBP $\beta$ immunostaining in astrocytes, identified by GFAP (Fig. 2d), thus supporting the specificity of LysMCre-induced recombination in microglia.

We were next interested in determining whether the absence of C/EBP $\beta$ affects microglial function. To this 

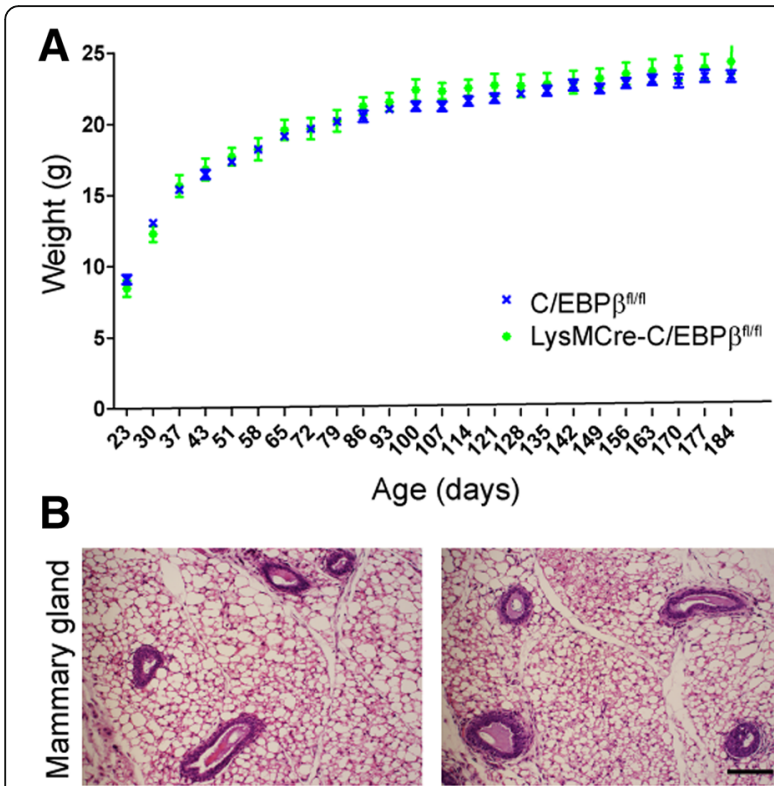

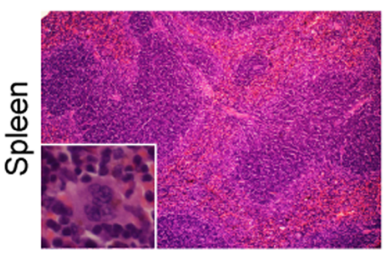

C/EBP $\beta^{\text {fl/fl }}$
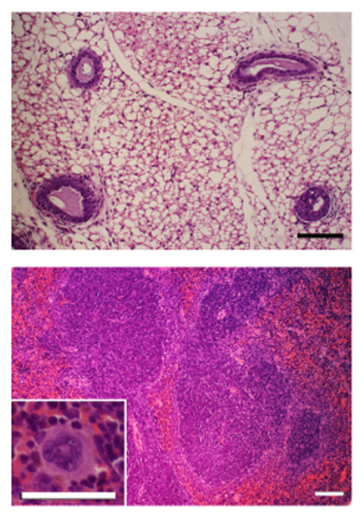

LysMCre-C/EBP $\beta^{\text {furf }}$
Fig. 1 Phenotypic characterization of LysMCre-C/EBP $\beta^{\text {fl/fl }}$ mice. a Body weight curves of female C/EBP $\beta^{\mathrm{fl} / \mathrm{fl}}(n=10)$ and LysMCre-C/ $\mathrm{EBP}^{\mathrm{fl} / \mathrm{fl}}(n=6)$ mice shown as mean \pm SEM. No significant differences in body weight are observed between both genotypes. b Representative microscopic images of haematoxylin and eosin staining of the mammary gland and spleen from 10-week-old C/EBP ${ }^{f / f l}$ and LysMCre-C/EBP $\beta^{f / / f l}$ mice. Normal ductal development and branching as well as unaltered adipose tissue in mammary gland from both genotypes are noted. Normal morphology of red and white pulps and megakaryocytes (insets) are also seen in both genotypes. No histological differences are observed also in the brain, lung, heart, liver, lung, kidney, femur bone and lumbar vertebrae (not shown). Scale bars $100 \mu \mathrm{m}$ (large images) and $50 \mu \mathrm{m}$ (insets)

end, we assessed NO production and NOS2 expression, activation-induced cell death (AICD) and phagocytosis of bacterial cells by primary microglial cultures from $\mathrm{C} /$ $E B P \beta^{\mathrm{fl} / \mathrm{fl}}$ and LysMCre-C/EBP $\beta^{\mathrm{fl} / \mathrm{fl}}$ mice. NO production is a key element in pro-inflammatory microglial activation and others, and we showed its regulation by $\mathrm{C} /$ $\mathrm{EBP} \beta$ in microglia $[3,24]$. In primary microglial cultures from $C / E B P \beta^{\mathrm{fl} / \mathrm{fl}}$ mice, co-treatment of LPS with IFNY induced a dose-dependent increase in $\mathrm{NO}$ production which was markedly attenuated in LysMCre-C/EBP $\beta^{\mathrm{fl} / \mathrm{fl}}$ microglia (Fig. 3a). We then assessed by immunocytochemistry the expression of NOS2, the NO producing enzyme in activated microglia, in mixed glial cultures from both genotypes in control and LPS + IFNY conditions. NOS2 was undetectable in control conditions, but it was induced by LPS + IFN $\gamma$ in $C / E B P \beta^{\mathrm{f} / / \mathrm{fl}}$ glial cultures, and this response was markedly attenuated in LysMCre-C/EBP $\beta^{\mathrm{f} / / \mathrm{ll}}$ cultures (Fig. 3b). CD11b immunostaining revealed the microglial nature of NOS2expressing cells in activated mixed glial cultures. In this experiment, we could not use Iba1 as microglial marker because both anti-Iba1 and anti-NOS2 antibodies were from a rabbit. These findings provide a first indication of an attenuated pro-inflammatory response in microglia from LysMCre-C/EBP $\beta^{\mathrm{fl} / \mathrm{fl}}$ mice.

Overactivation of microglia leads to apoptotic cell death in a process named AICD thought to contribute to maintaining low numbers of activated microglia after brain damage [25]. The role of C/EBP $\beta$ in microglial AICD has not been studied, but since $C / E B P \beta$ regulates microglial $\mathrm{NO}$ production and $\mathrm{NO}$ is an important AICD effector in microglia, we hypothesized that AICD could be attenuated in C/EBP $\beta$-deficient microglia. In $\mathrm{C} /$ $\mathrm{EBP} \beta^{\mathrm{fl} / \mathrm{fl}}$ microglia, LPS induced a clear AICD effect $(40 \%$ cell death, 5 days) that was markedly potentiated by IFN $\gamma$ (90\% cell death, 5 days) (Fig. 3c). The same effect was observed in LysMCre-C/EBP $\beta^{\mathrm{H} / \mathrm{fl}}$ microglia thus ruling out a key role for C/EBP $\beta$ in microglial AICD. Similar results were obtained 3 and 7 days after treatments (data not shown).

We were finally interested to study the role of C/EBP $\beta$ in phagocytosis, one of the major physiological roles of microglia. To this end, control and activated microglia from both genotypes were incubated with autofluorescent S. typhimurium, a Gram-negative bacterium. The percentage of infected microglia and the number of bacteria per microglial cell were assessed $30 \mathrm{~min}$ and $4 \mathrm{~h}$ after addition of bacteria. Data obtained in $30 \mathrm{~min}$ indicate phagocytic capacity whereas data in $4 \mathrm{~h}$ show the ability of microglia to eliminate phagocytosed bacterial cells. As shown in Fig. 3d, 30 min after bacteria addition, no differences in phagocytosis between both genotypes were observed in untreated microglia. However, after LPS + IFN $\gamma$ treatment, LysMCre-C/EBP $\beta^{\mathrm{fl} / \mathrm{fl}}$ microglia showed higher number of infected cells and higher intake of bacteria per cell than $C / E B P \beta^{\text {fl/fl }}$ microglia, whereas after LPS treatment, LysMCre-C/EBP $\beta^{\mathrm{f} / \mathrm{fl}}$ microglia showed higher number of phagocytosed bacteria per cell. Four hours after bacteria addition, differences between both genotypes were attenuated. Only in LysMCre-C/EBP $\beta^{\mathrm{f} / \mathrm{fl}}$ microglia treated with LPS + IFN $\gamma$ there was an increase in the percentage of infected cells, but of lower magnitude than that observed in $30 \mathrm{~min}$. In $\mathrm{C} / \mathrm{EBP} \beta^{\mathrm{fl} / \mathrm{fl}}$ microglia, the percentage of infected cells and the number of bacteria/cell in $4 \mathrm{~h}$ were not decreased when compared with the 30 min values, with only one exception (\% infected cells after LPS treatment). In contrast, in LysMCre-C/EBP $\beta^{\mathrm{fl} / \mathrm{fl}}$ microglia, the percentage of infected cells and the number of 


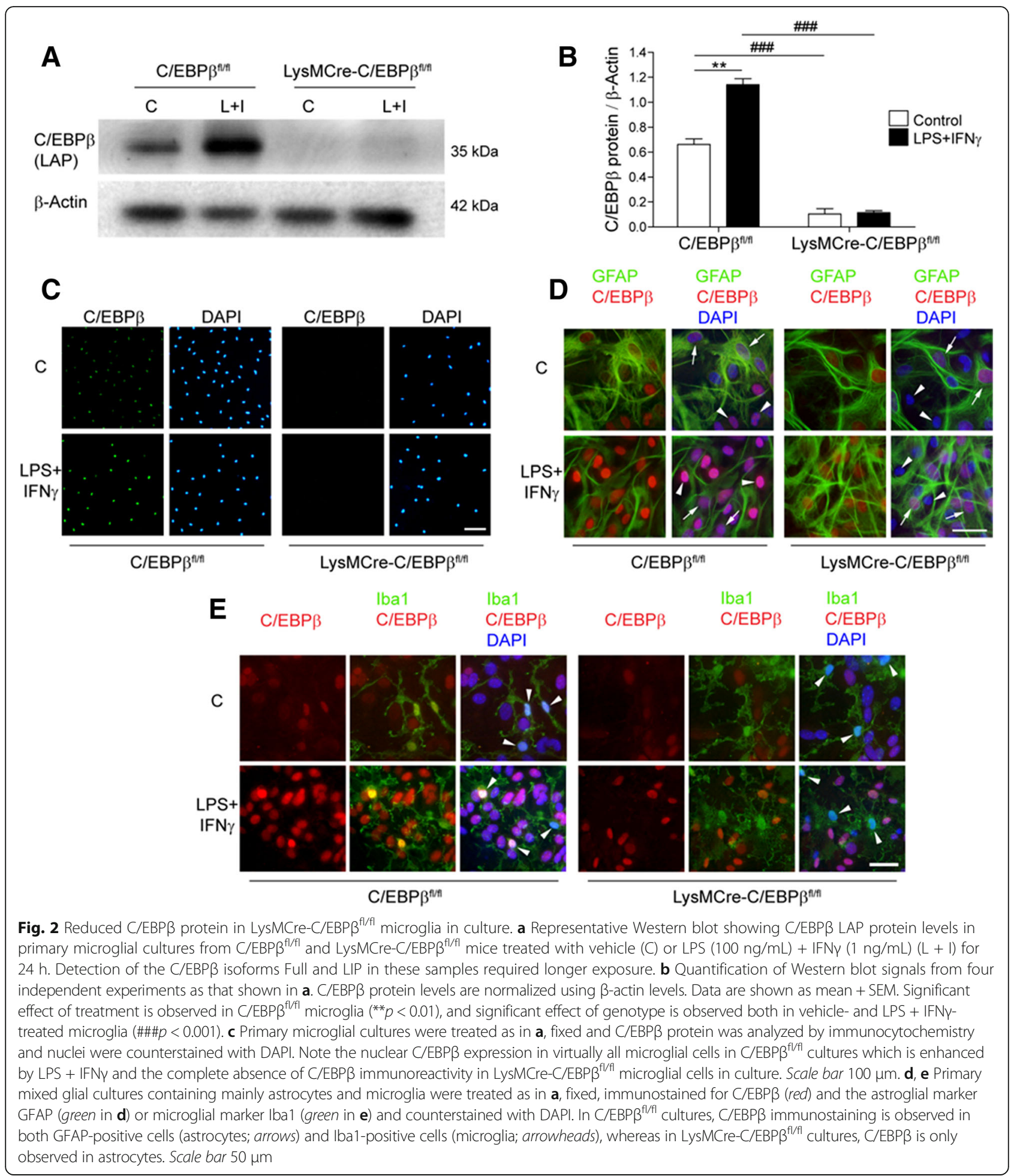

bacteria/cell in $4 \mathrm{~h}$ were decreased vs $30 \mathrm{~min}$ (with one exception: bacteria/cell after LPS + IFN $\gamma$ treatment). These data demonstrate increased phagocytic activity and higher capacity to eliminate phagocytosed bacteria of $\mathrm{C} / \mathrm{EBP} \beta$-deficient microglia.

\section{C/EBP $\beta$-deficient cultured microglia show marked} changes of gene expression

After demonstrating the widespread deficiency of $\mathrm{C} /$ $E B P \beta$ in microglia from LysMCre-C/EBP $\beta^{\mathrm{fl} / \mathrm{fl}}$ mice and its functional effects, we assessed the global effects of 


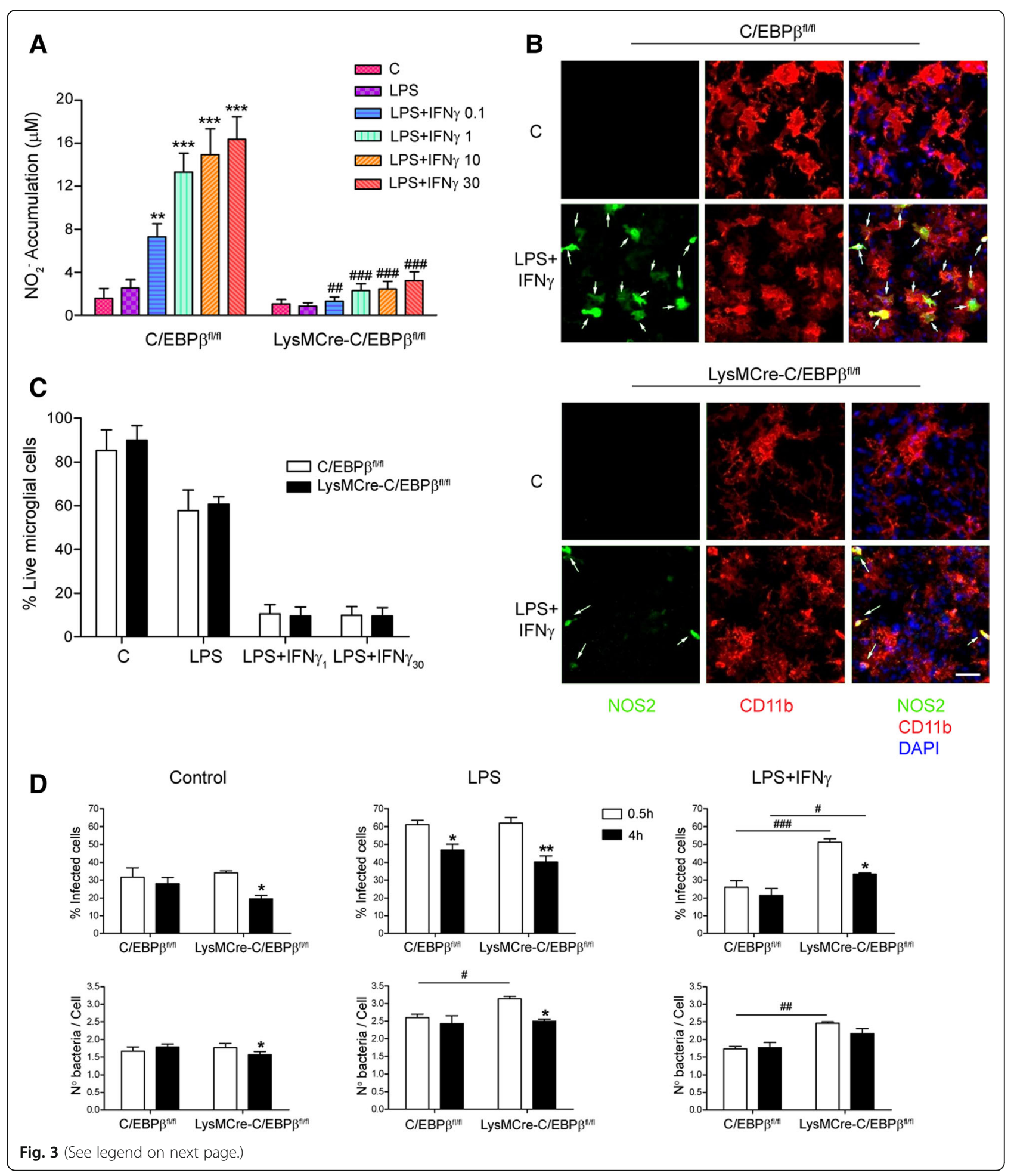




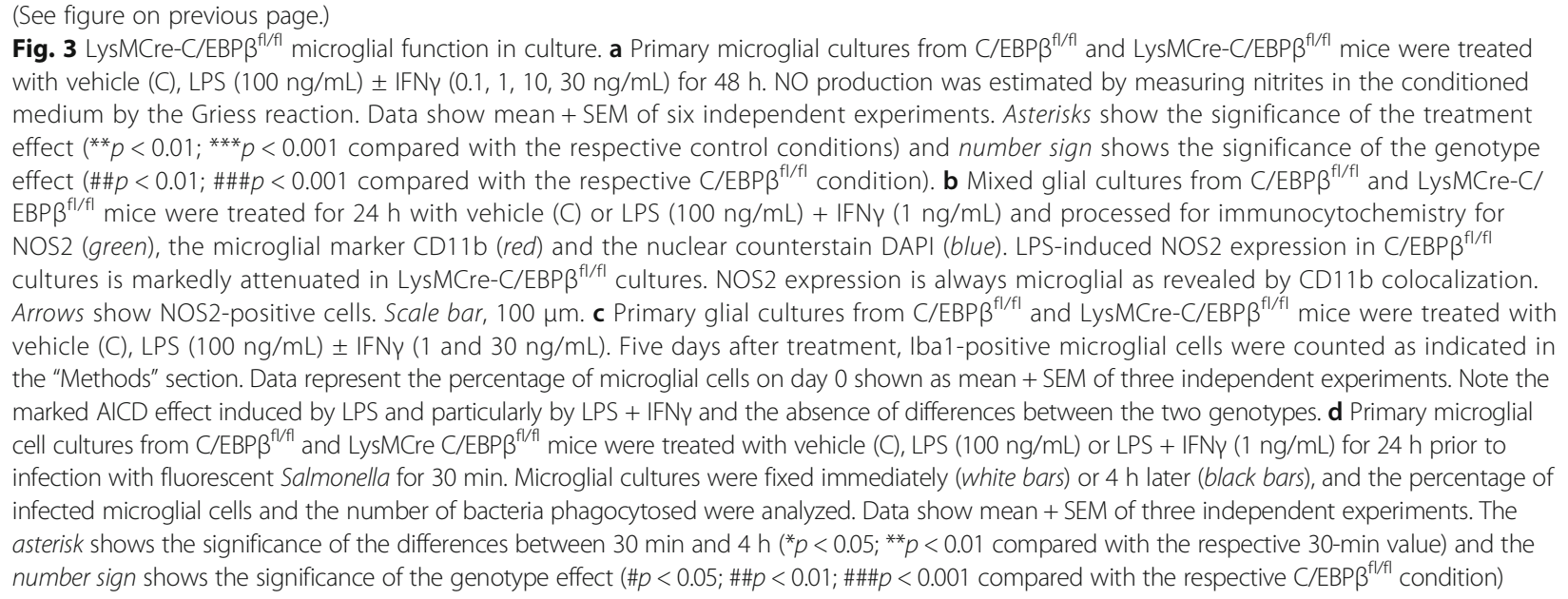

$\mathrm{C} / \mathrm{EBP} \beta$ deficiency in microglial gene expression by comparing by RNAseq the transcriptomic profiles of $\mathrm{C} / \mathrm{EBP} \mathrm{f}^{\mathrm{fl} / \mathrm{fl}}$ and LysMCre-C/EBP $\beta^{\mathrm{fl} / \mathrm{fl}}$ primary microglial cultures treated with vehicle, LPS or LPS + IFN $\gamma$ for $6 \mathrm{~h}$.

RNA libraries and sequencing where prepared as described in the "Methods" section. Unsupervised cluster analysis using normalized counts (RPKM) showed a strong effect of treatments on microglial RNA expression. The 21 samples were grouped in three clusters corresponding without exception to microglial cultures treated with vehicle $(n=7)$, LPS $(n=7)$ and LPS + IFNy $(n=7)$ (Fig. 4a). The clustering analysis also revealed a marked effect of genotype on RNA expression, particularly in activated microglia. Thus, LPS- and LPS + IFN $\gamma$-treated samples were subclassified into two clusters corresponding to genotypes $\mathrm{C} / \mathrm{EBP} \beta^{\mathrm{fl} / \mathrm{fl}}$ and LysMCre-C/EBP $\beta^{\mathrm{fl} / \mathrm{fl}}$ microglia (Fig. 4a). This analysis strongly suggests that $C / E B P \beta$ plays an important role in the regulation of gene expression in activated microglia. Afterwards, the validated pipeline for differentially expressed genes (DEGs) detection Rsubread/ voom normalization/limma [20] was used selecting genes that complied with fold change $>2$ and $p<0.01$ (Benjamini-Hochberg method for multiple comparisons) in pairs of conditions compared. Out of 15228 genes with significant RNA expression in microglia, 1068 were affected by $C / E B P \beta$ deficiency. As the Venn diagram in Fig. $4 \mathrm{~b}$ shows, most of these were genes also significantly affected by LPS (717 genes) or LPS + IFNY (708 genes) and only 201 of $\mathrm{C} / \mathrm{EBP} \beta$-dependent genes were unaffected by LPS or LPS + IFN $\gamma$ again indicating a major role for $C / E B P \beta$ on gene expression in activated microglia. In addition, we separately analyzed the samples to show the DEG between $\mathrm{C} / \mathrm{EBP} \beta^{\mathrm{fl} / \mathrm{fl}}$ and LysMCre-C/EBP $\beta^{\mathrm{fl} / \mathrm{fl}}$ microglia in each treatment condition (Control, LPS, LPS + IFN $\gamma$ ). In control conditions $\mathrm{C} / \mathrm{EBP} \beta$ absence resulted in more genes being downregulated (44 genes) than upregulated (13 genes); in the LPS condition the opposite was observed
(459 upregulated and 339 downregulated); and in the LPS + IFN $\gamma$ condition the number of upregulated and downregulated genes was similar (197 and 217 genes, respectively) (Additional file 1: Tables $\mathrm{S} 1-\mathrm{S} 6$ ).

DEGs $\log 2$ values of $C / E B P \beta^{\mathrm{fl} / \mathrm{fl}}$ and LysMCre-C/EBP $\beta^{\mathrm{fl} / \mathrm{fl}}$ comparison $(n=1068)$ were normalized to be expressed in standard deviation values $( \pm 3 \mathrm{SD})$ and represented on a heatmap (Fig. 4c). A hierarchical cluster algorithm for samples and genes revealed two sample clusters: cluster 1 which included untreated samples of both genotypes and treated LysMCre-C/EBP $\beta^{\mathrm{fl} / \mathrm{fl}}$ samples and cluster 2 which included treated $C / E B P \beta^{\text {fl/fl }}$ samples. This shows that LPS \pm IFN $\gamma$-treated LysMCre-C/EBP $\beta^{\text {fl/fl }}$ microglia have an attenuated phenotype with a gene expression profile closer to untreated microglia than to treated $\mathrm{C} /$ $\mathrm{EBP} \beta^{\mathrm{fl} / \mathrm{fl}}$ microglia. This analysis also shows a neat separation of untreated samples into two clusters corresponding to the two genotypes demonstrating in an unbiased way the altered biology of $\mathrm{C} / \mathrm{EBP} \beta$-deficient microglia in basal conditions. Additionally, a Weighted Correlation Gene Network Analysis (WGCNA) was performed, separating all 15,228 genes into 35 modules. The dendrogram of WGCNA revealed a large grouping of genes corresponding to module "turquoise" which represent genes with decreased expression upon LPS or LPS + IFN $\gamma$ treatments (Additional file 2: Figure S1A). Complementarily, dendrogram clustering of modules highlighted module "violet" as the closest group of genes correlating with genotype differences (Additional file 2: Figure S1B). Module violet contained $\mathrm{C} / \mathrm{EBP} \beta$ as expected (Additional file 2: Figure S1C) and additionally a set of genes linked by outer literature databases (Metacore ${ }^{\mathrm{Tm}}$; Additional file 2: Figure S1D) and within the distance measures of experiment data (Additional file 2: Figure S1E).

Finally, we used the Genecodis platform [21] to obtain DEG enrichment in different annotation databases, specifically for Gene Ontology (GO) terms for Biological 


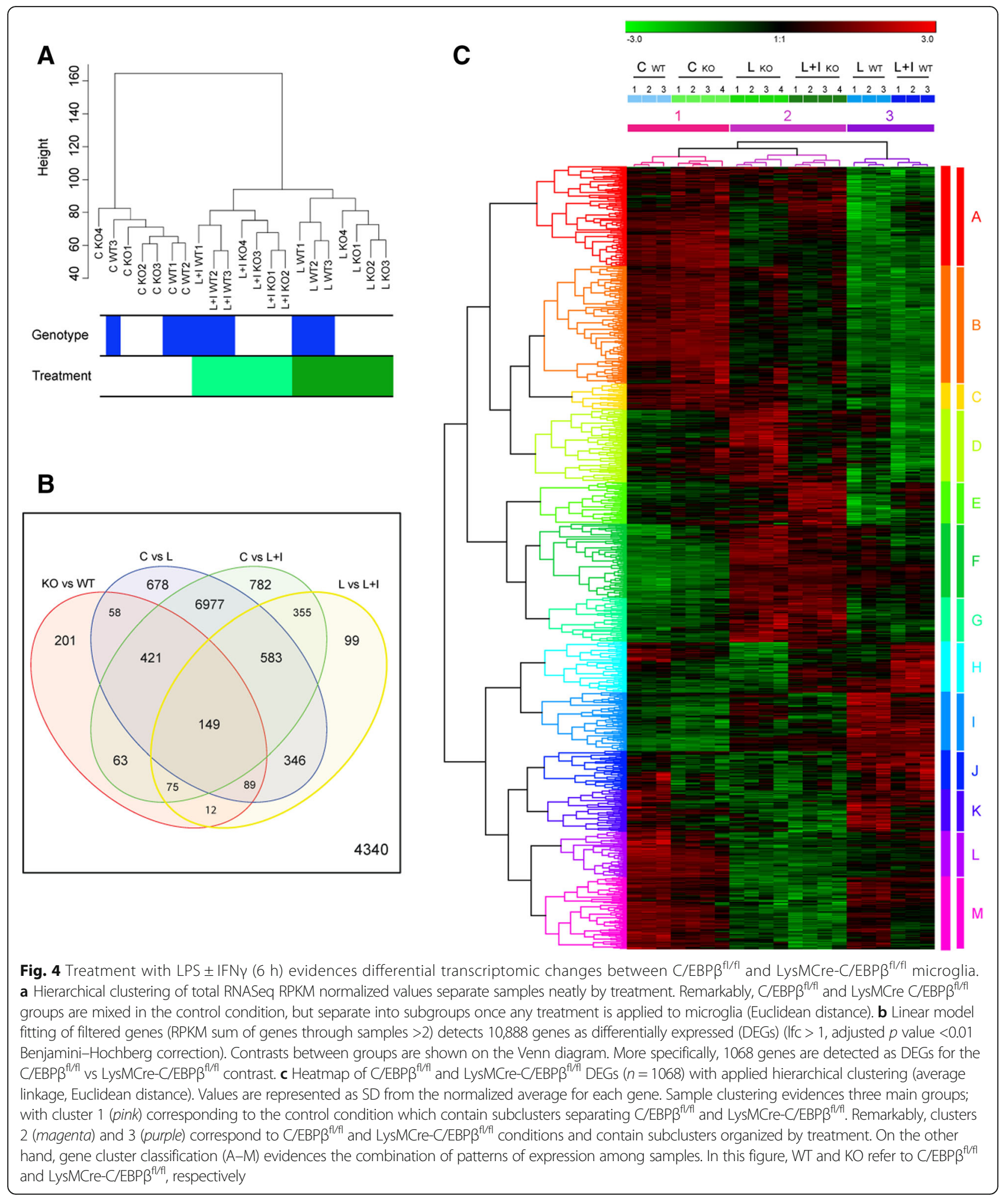

Process (Fig. 5a), and for the Kyoto Encyclopedia of Genes and Genomes (KEGG) pathways (Fig. 5b). Notably, various GO categories and KEGG pathways related to inflammatory and innate immune responses were among the most significantly represented (e.g. inflammatory response, GO:0006954; Fig. 5c). Interestingly, lysosome and phagosome KEGG pathways were also particularly represented (Fig. 5d-f). 


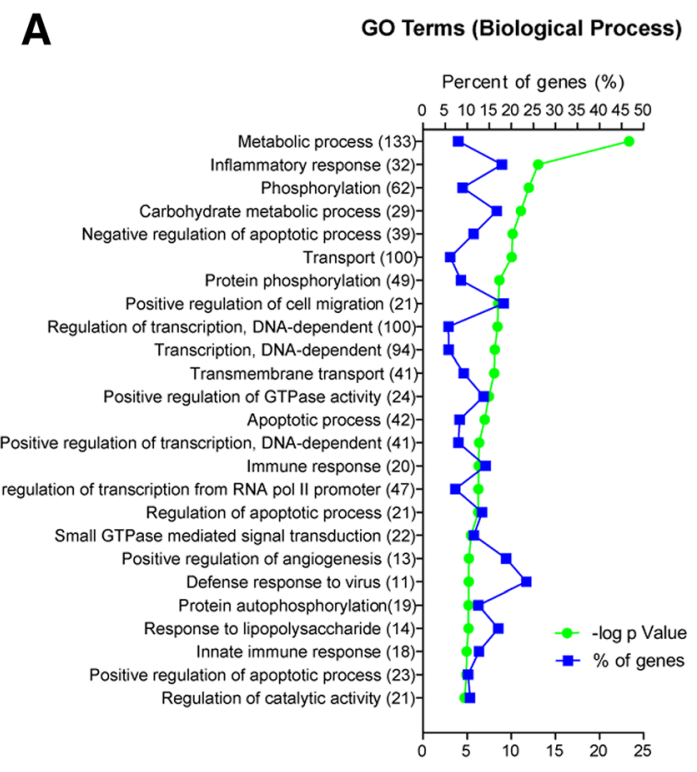

-log corrected $p$ Value

C Inflammatory response (GO:0006954)

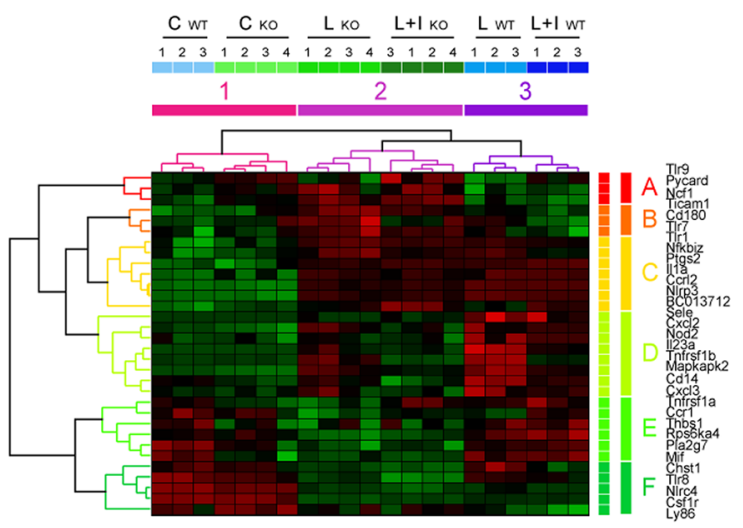

E

Regulation of actin cytoskeleton (Kegg:04810)

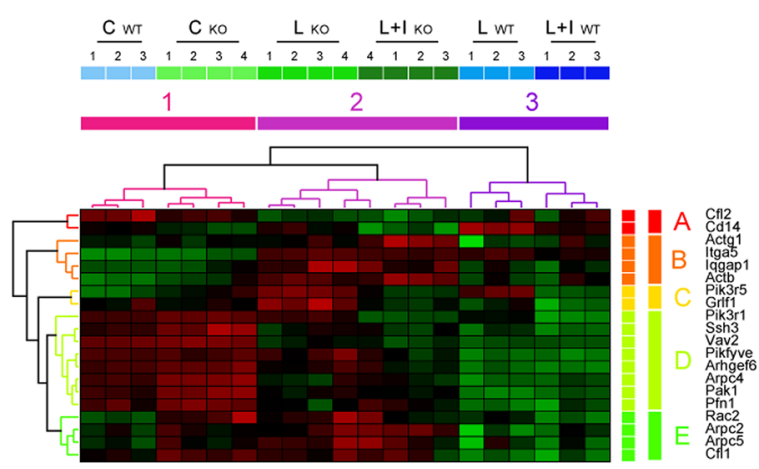

B

KEGG Pathway

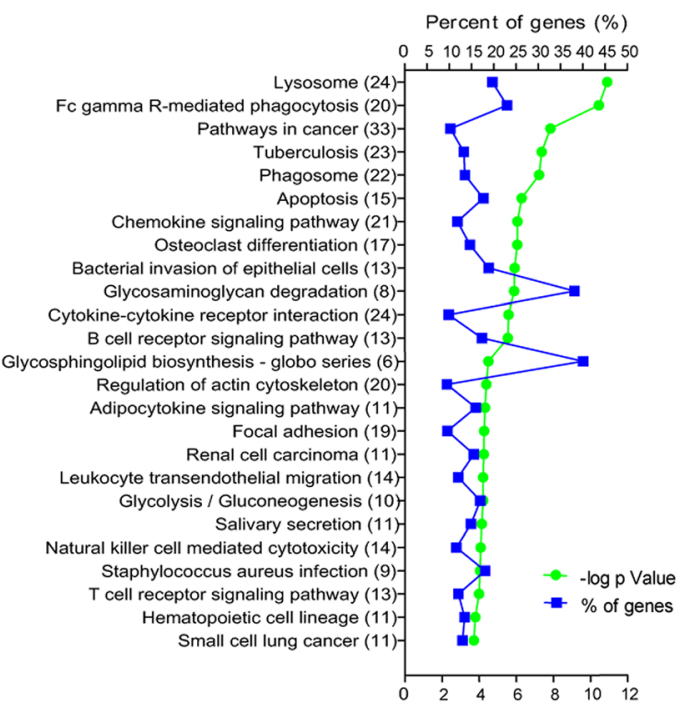

$-\log$ Corrected $p$ Value

\section{Phagosome (Kegg:04145)}

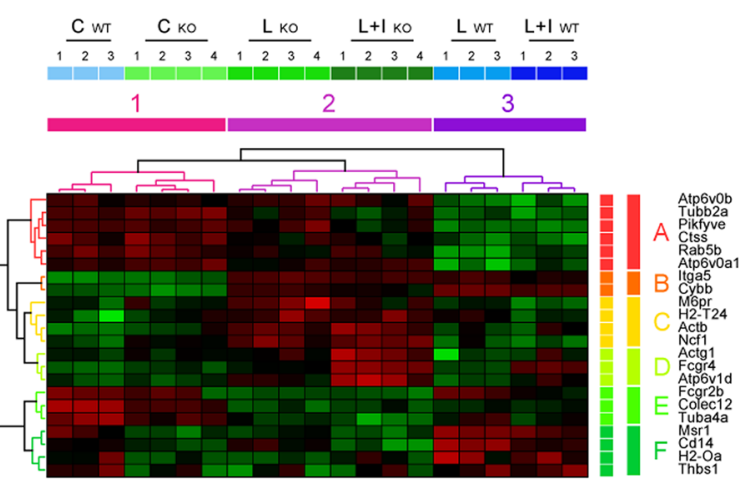

$\mathbf{F}$

Lysosome (Kegg:04142)

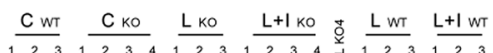

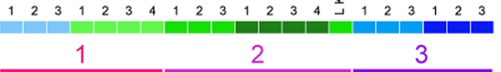
$\sqrt{4 \sqrt{4}}$

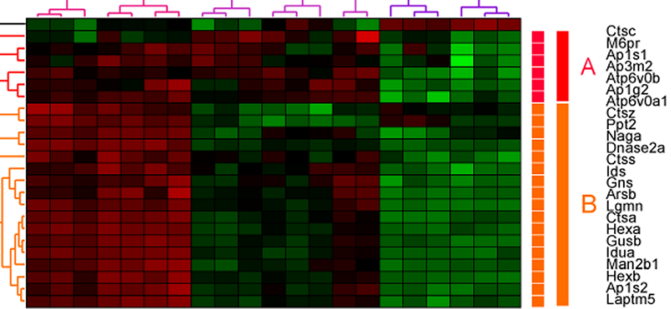

Fig. 5 (See legend on next page.) 


\section{(See figure on previous page.)}

Fig. 5 Ontology enrichment analyses reveal biological implication of obtained DEGs with microglial processes. C/EBP $\beta^{\mathrm{fl} / \mathrm{fl}}$ and LysMCre-C/EBP $\beta^{\mathrm{fl} / \mathrm{fl}}$ DEGs where fed into the Genecodis platform for ontology enrichment. a $p$ value and gene percentage annotation graph of the biological process (BP) category from Gene Ontology database (hypergeometric distribution). b $p$ value and gene percentage annotation graph from Kyoto Encyclopedia of Genes and Genomes (KEGG) pathway enrichment analyses (hypergeometric distribution). c-e Heatmap of genes annotated with the enriched categories shown above (hierarchical clustering, average linkage, Euclidean distance, scale in SD over normalized gene expression in log2 values). Note the existence of gene clusters with distinct patterns of gene expression changes, e.g. cluster B in $\mathbf{f}$ corresponds to lysosome-related genes with reduced expression upon LPS \pm IFNy treatments, in C/EBP $\beta^{\mathrm{fl} / \mathrm{fl}}$ microglia that is attenuated in LysMCre-C/ $E B P \beta^{f l / f l}$ microglia. In this figure, WT and KO refer to $C / E B P \beta^{f l / f l}$ and $L y s M C r e-C / E B P \beta^{f l / f l}$, respectively

In summary, this analysis shows the importance of microglial $C / E B P \beta$, since its deletion affects the expression of 1068 genes. It confirms the involvement of $C / E B P \beta$ in the regulation of pro-inflammatory programs in activated microglia, but it also reveals a role of C/EBP $\beta$ in other programs such as phagocytosis and apoptosis. Interestingly, it reveals for the first time a role for $C / E B P \beta$ in nonstimulated microglia.

\section{Robust C/EBP $\beta$ deletion in LysMCre-C/EBP $\beta^{\text {fl/fl }}$ microglia in vivo and subsequent attenuated pro-inflammatory gene expression}

We were next interested to study the consequences of microglial $\mathrm{C} / \mathrm{EBP} \beta$ deletion in vivo. A first step was to estimate the efficiency of LysMCre-induced recombination of C/EBP $\beta$ LoxP sites in microglial cells in vivo. To this end, microglia was acutely isolated by immunomagnetic separation from the whole brain of $C / E B P \beta^{\mathrm{fl} / \mathrm{fl}}$ and LysMCre-C/EBP $\beta^{\mathrm{fl} / \mathrm{fl}}$ adult mice treated systemically with LPS or vehicle for $16 \mathrm{~h}$. At the dose and time frame used, systemic LPS induces a neuroinflammatory response with increased $C / E B P \beta$ expression in the CNS [26]. C/EBP $\beta$ protein was not detected by Western blot in microglia protein extracts from vehicle-treated mice of both genotypes. Systemic LPS induced the strong expression of C/EBP $\beta$ Full and LAP protein isoforms in $\mathrm{C} /$ $E B P \beta^{\mathrm{fl} / \mathrm{fl}}$ microglia, and this induction was completely ablated in microglia isolated from LPS-treated LysMCre$\mathrm{C} / \mathrm{EBP} \beta^{\mathrm{fl} / \mathrm{fl}}$ mice brains (Fig. 6a, b). These findings were confirmed and extended by immunocytochemistry for $\mathrm{C} / \mathrm{EBP} \beta$ in cytospin microglial preparations. Immunostaining for the microglial markers CD68 (Fig. 6c) and Iba1 (not shown) confirmed the microglial nature of the ex vivo isolated cells. As shown in Fig. 6c, d, C/EBP $\beta$ immunoreactivity was not observed in microglia from vehicle-treated mice of both genotypes, whereas it was present in virtually $100 \%$ of microglial cells from LPStreated $C / E B P \beta^{\mathrm{fl} / \mathrm{fl}}$ mice. In these cells, $\mathrm{C} / \mathrm{EBP} \beta$ showed nuclear localization. In contrast, in LPS-treated LysMCre$\mathrm{C} / \mathrm{EBP} \beta^{\mathrm{fl} / \mathrm{fl}}$ microglial cytospin preparations, $\mathrm{C} / \mathrm{EBP} \beta$ immunoreactivity was only observed in $11 \pm 1 \%$ of microglial cells (Fig. 6c, d). These findings demonstrate a robust $\mathrm{C} /$ $E B P \beta$ deletion in LysMCre-C/EBP $\beta^{\mathrm{fl} / \mathrm{fl}}$ microglial cells not only in vitro, where the efficiency of recombination approaches $100 \%$, but also in vivo where this efficiency approaches $90 \%$. These results support the use of LysMCre-C/EBP $\beta^{\mathrm{fl} / \mathrm{fl}}$ mice as a model to study the functional effects of microglial $C / E B P \beta$ in vitro and in vivo.

To investigate the role of $C / E B P \beta$ on gene expression in activated microglia in vivo, we isolated microglia from the brains of vehicle or LPS-treated $(16 \mathrm{~h}) \mathrm{C} / \mathrm{EBP} \beta^{\mathrm{fl} / \mathrm{fl}}$ and LysMCre-C/EBP $\beta^{\mathrm{fl} / \mathrm{fl}}$ mice, and the expression of selected genes was assessed by quantitative real-time PCR (qRT-PCR). For this analysis, we included the proinflammatory cytokines Tnf, Il23a and Csf3 and the key enzymes in the production of the inflammatory mediator prostaglandin E2 (Ptges) and the free radical superoxide anion $(\mathrm{Cybb})$. These are genes with a prominent role in microglial activation whose expression in cultured microglia was significantly dependent upon C/EBP $\beta$ according to RNAseq data. We first confirmed that the expression of these genes was affected by $C / E B P \beta$ by analyzing their expression by qRT-PCR in a new series of microglial cultures independent from those used to generate RNAseq data. As shown in Fig. 7 (insets), C/EBP $\beta$ deficiency resulted in decreased LPS- and/or LPS + IFN $\gamma$-induced expression of these genes in cultured microglia. Interestingly, in vivo expression of these five genes was upregulated by systemic LPS in microglia, and in all cases, these increases were significantly blunted in microglia from LysMCre-C/EBP $\beta^{\mathrm{fl} / \mathrm{fl}}$ mice (Fig. 7). These results demonstrate for the first time that the specific absence of $C / E B P \beta$ in microglia in vivo results in attenuated expression of pro-inflammatory genes.

\section{Deletion of C/EBP $\beta$ in myeloid cells is protective in an animal model of multiple sclerosis}

In order to test whether the myeloid-specific $\mathrm{C} / \mathrm{EBP} \beta \mathrm{ab}$ sence and the resulting attenuation of microglial activation have neuroprotective effects in vivo, we induced EAE, an animal model of multiple sclerosis, in LysMCre-C/EBP $\beta^{\mathrm{fl} / \mathrm{fl}}$ mice. We first analyzed the time course of $C / E B P \beta$ expression changes in EAE in three spinal cord and three brain regions of wild-type mice. Figure $8 \mathrm{a}$, b shows the results obtained in the thoracic spinal cord and hindbrain. Similar findings were obtained in the cervical and lumbar spinal cord and in the midbrain and forebrain (data not shown). EAE was associated with increased C/EBP $\beta$ mRNA levels 


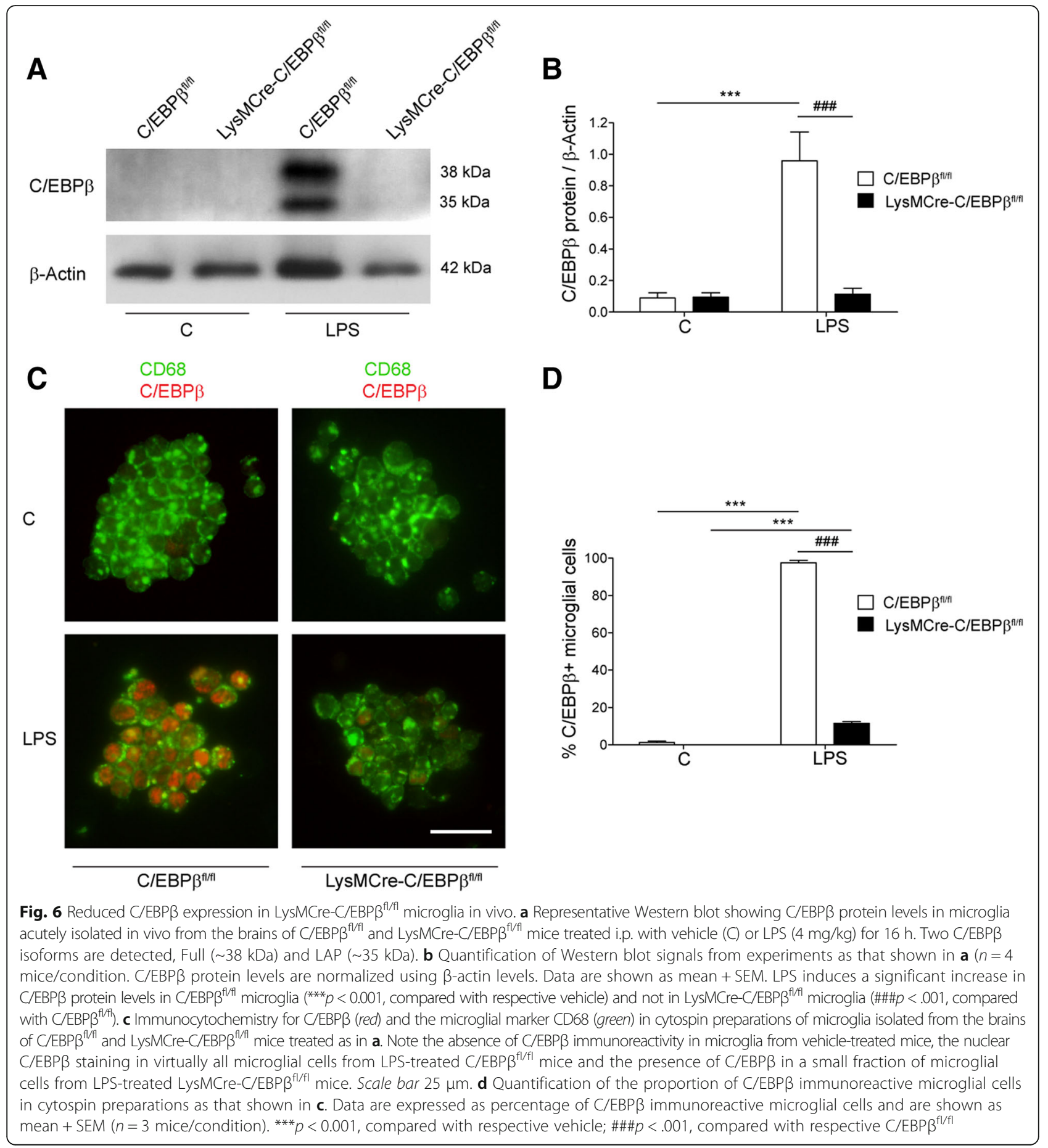

in all regions analyzed. The most marked increase was observed on day 14. A moderate increase on days 21 and 28 was observed in some of the CNS regions analyzed, such as the thoracic spinal cord (Fig. 8a, b). To study whether these mRNA changes were also observed at the protein level, a time course analysis of C/EBP $\beta$ protein expression was performed in thoracic spinal cord by Western blot. As shown in Fig. 8c, d, a marked increase in $\mathrm{C} / \mathrm{EBP} \beta$ protein levels was observed on day 14, in correspondence with the mRNA data. Out of the three C/EBP $\beta$ isoforms, LAP showed the highest expression in these samples.

We next analyzed the clinical course of EAE in LysMCre-C/EBP $\beta^{\mathrm{fl} / \mathrm{fl}}$ mice and compared it with that of the two parent lines, LysMCre and $\mathrm{C} / \mathrm{EBP} \beta^{\mathrm{fl} / \mathrm{fl}}$. Both parent lines showed a very similar course of the disease. Mice started to develop multiple sclerosis-like symptoms 


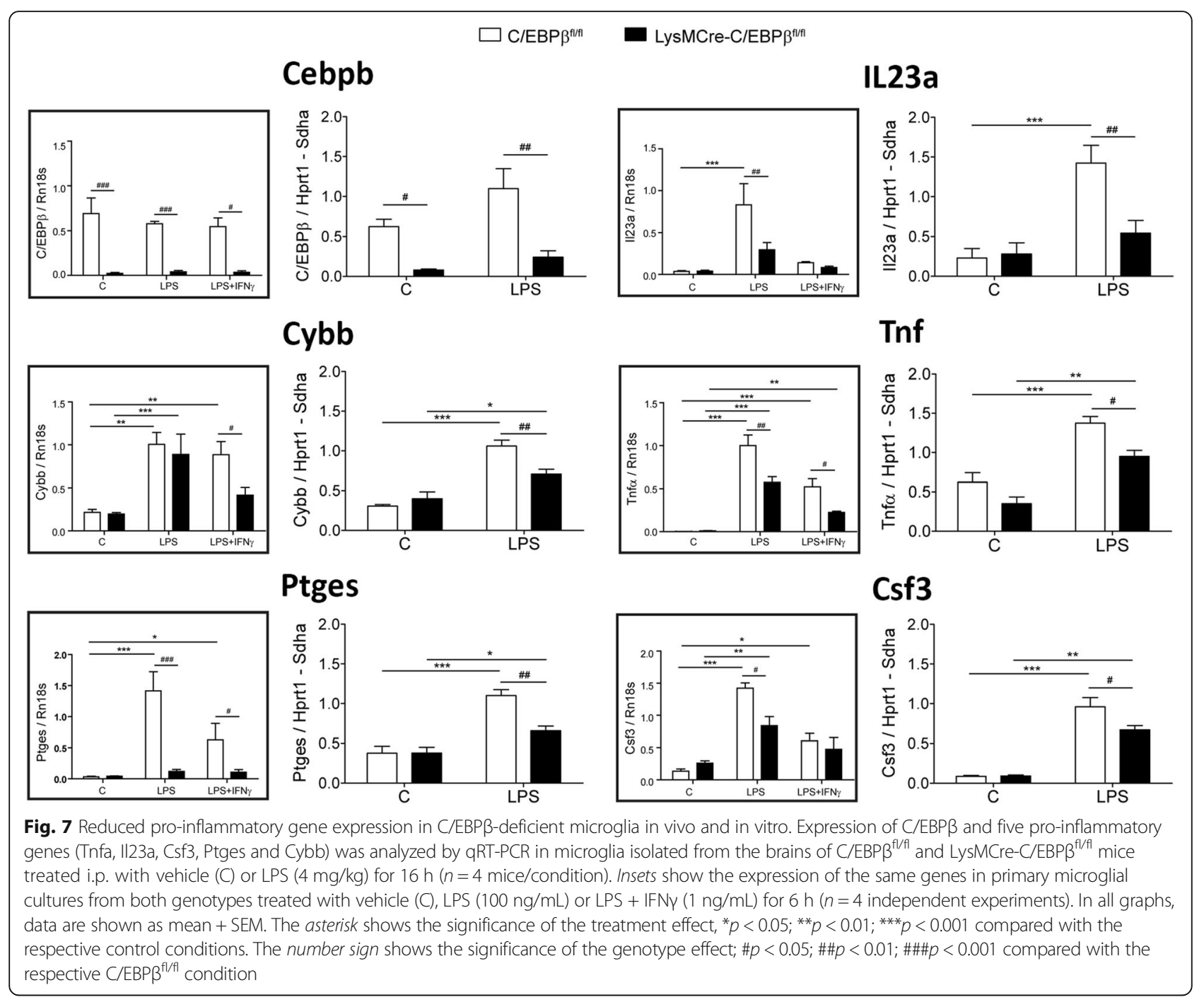

10 days after immunization. These symptoms progressed steadily, peaked on day 20 and then plateaued until the end of the experiment on day 52. In contrast, LysMCre-C/ $\mathrm{EBP} \mathrm{\beta}^{\mathrm{fl} / \mathrm{fl}}$ mice showed attenuated EAE symptoms with a milder initial phase, a lower score at the peak of the disease and a partial recovery phase (Fig. 8e). Since no differences were observed between both parent lines, this experiment was repeated twice using only $\mathrm{C} / \mathrm{EBP} \beta^{\mathrm{fl} / \mathrm{fl}}$ mice as the control. In both experiments, LysMCre-C/EBP $\beta^{\mathrm{fl} / \mathrm{fl}}$ mice showed a highly significant reduction in EAE score with a similar profile as that of experiment 1 (data not shown). The incidence of EAE in the three experiments was similar for both genotypes (73\% (30/41) for C/EBP $\beta^{\mathrm{fl} /}$ ${ }^{\mathrm{fl}}$ mice and $68 \%(26 / 38)$ for LysMCre-C/EBP $\beta^{\mathrm{f} / \mathrm{fl}}$ mice), and mortality was higher in $\mathrm{C} / \mathrm{EBP} \beta^{\mathrm{fl} / \mathrm{fl}}$ mice $(12 \% ; 5 / 41)$ than in LysMCre-C/EBP $\beta^{\mathrm{f} / \mathrm{fl}}$ mice $(5 \% ; 2 / 38)$.

Finally, C/EBP $\beta$ expression was studied in human multiple sclerosis. To this end, $\mathrm{C} / \mathrm{EBP} \beta$ protein levels were analyzed by Western blot in temporal cortex postmortem samples from multiple sclerosis and non-neurological controls. As shown in Fig. 8f, g, all three C/EBP $\beta$ isoforms were detected, LAP being the most expressed. Interestingly, protein levels of Full and LAP, the two C/EBP $\beta$ activator isoforms, were increased in multiple sclerosis samples by $55 \%$ $(p<0.05)$ and $93 \%(p<0.001)$, respectively.

\section{Discussion}

C/EBP $\beta$-deficient mice show reduced neuronal damage induced by excitotoxic or ischemic insults in vivo $[7,9]$. Since C/EBP $\beta$ is expressed by many cell types, the question of which is the cell type(s) responsible for the neuroprotective effects of $\mathrm{C} / \mathrm{EBP} \beta$ absence in these models is of interest. In neuron-microglia co-cultures, the absence of $\mathrm{C} / \mathrm{EBP} \beta$ only in microglial cells completely abrogates their neurotoxic effects elicited by LPS + IFN $\gamma$ activation [3]. This led us to hypothesize that C/EBP $\beta$ inhibition in the microglia could have therapeutic potential as a target to attenuate deleterious effects of neuroinflammation. As a 
A

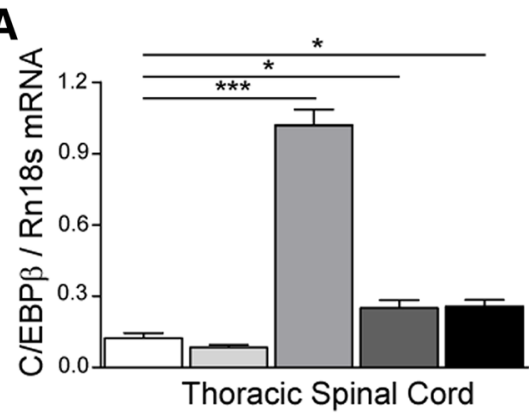

C

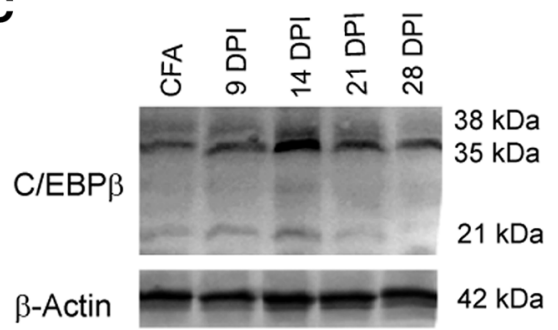

B

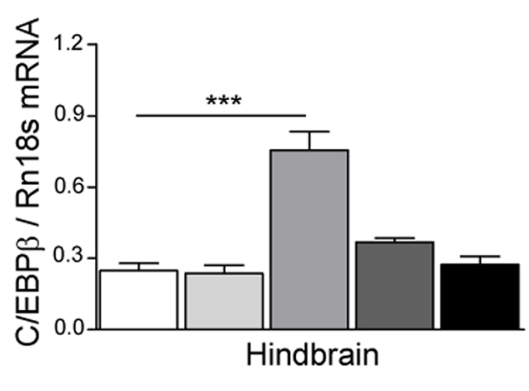

D

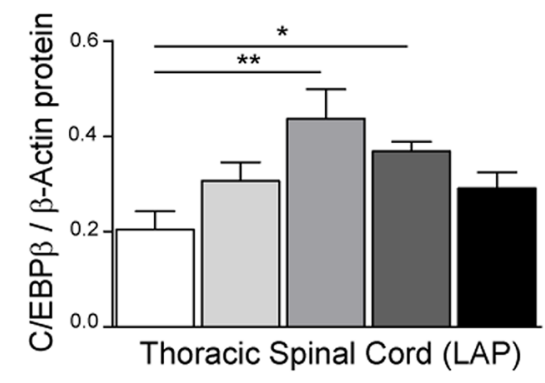

E

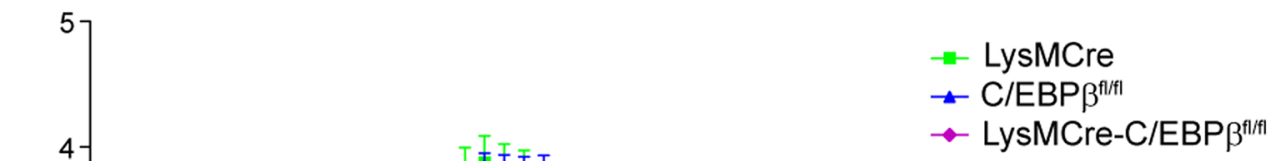

$28 \mathrm{DPI}$

9 DPI

$14 \mathrm{DPI}$

$21 \mathrm{DPI}$

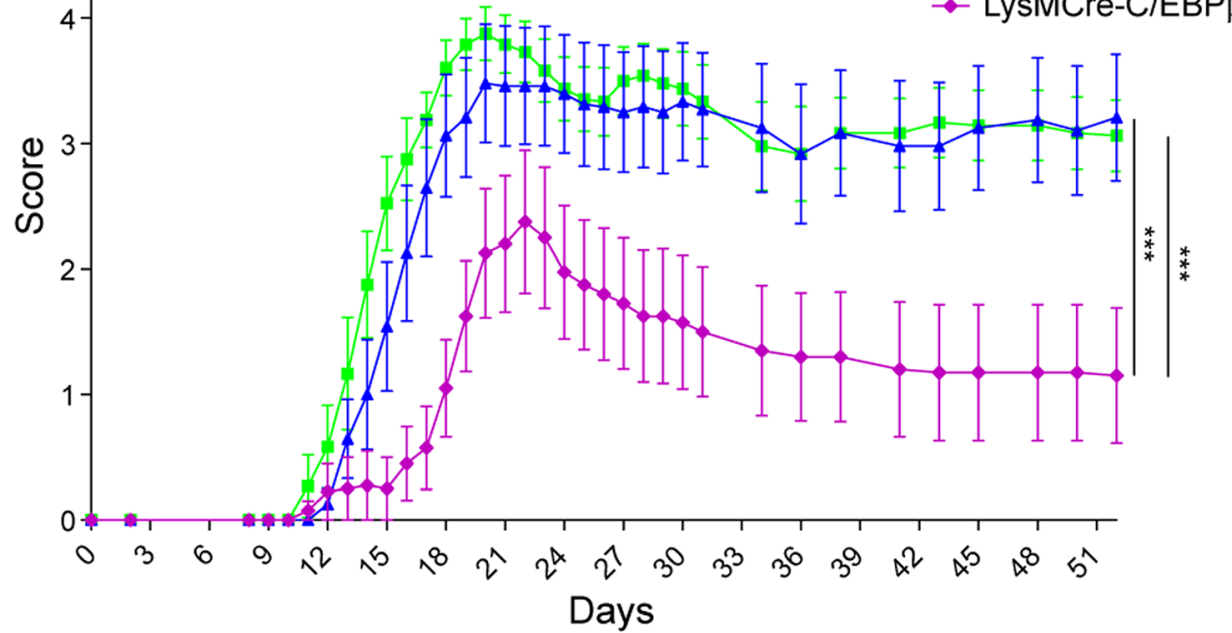

$\mathbf{F}$

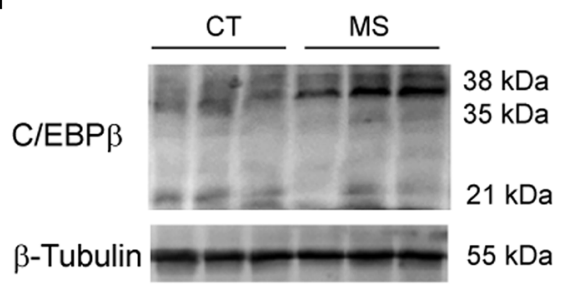

G

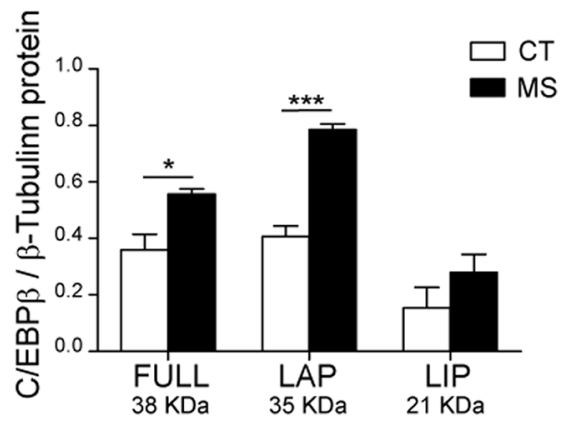

Fig. 8 (See legend on next page.) 


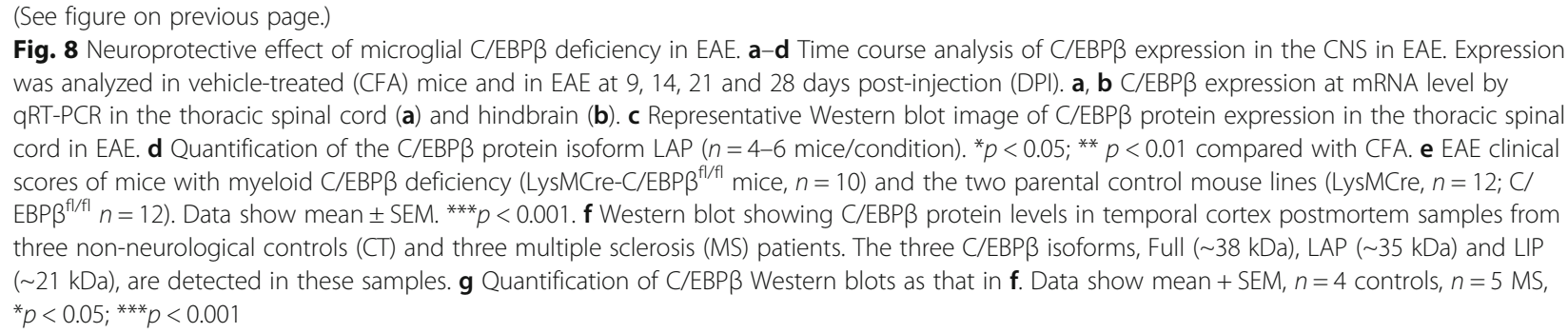

proof of concept, we have generated mice with $\mathrm{C} / \mathrm{EBP} \beta$ deficiency in myeloid cells. The results here presented show a marked attenuation of clinical symptoms of EAE in these mice. Besides, RNAseq analysis of cultured microglia shows that $C / E B P \beta$ plays a key role in the regulation of gene transcription in microglial activation and provides possible explanations for the neuroprotective effects of $\mathrm{C} /$ EBP $\beta$ absence in myeloid cells.

In order to produce $\mathrm{C} / \mathrm{EBP} \beta$ deletion in microglia, we have used the Cre-LoxP approach with Cre expression under the control of the LysM promoter [27]. Unlike astrocytes, for which GFAP promoter is considered the gold standard, there is not an ideal promoter to drive Cre expression in microglia. Promoters of LysM, CD11b, F4/ 80, CSF1 receptor or Iba1 genes have been used successfully, and in the last few years, CX3CR1 is becoming the most widely used promoter in this respect (reviewed by [28]), but in all cases, Cre expression is induced not only exclusively in microglia but also in other subsets of myeloid cells. The LysMCre mouse line has been used extensively to induce Cre expression in myeloid cells, particularly in macrophages and microglia. Data from crossing LysMCre mice with reporter mice have shown functional Cre in $30-45 \%$ of microglial cells in control CNS $[29,30]$ and strong recombination in primary microglial cultures [30-32]. Various studies have obtained positive results by using LysMCre in in vivo models that have been attributed primarily to microglia [33-35] or to microglia and macrophages [31, 36]. Our data clearly show an efficiency of recombination of LysMCre close to 100 and $90 \%$ in primary microglial cultures and in microglia in vivo in LPS-treated mice, respectively, supporting the use of this mouse line to drive Cre expression in microglia in these experimental models. In our opinion, the most likely interpretation of the neuroprotective effects of LysM-driven $C / E B P \beta$ deletion in EAE is that the absence of $C / E B P \beta$ in microglia results in an attenuated neuroinflammatory response, milder neurodegeneration and less severe EAE symptoms. However, macrophages and granulocytes show also LysM-driven Cre expression in this mouse line [27], and in both cell types, C/EBP $\beta$ expression has been reported [37]. We cannot therefore discard that C/EBP $\beta$ deletion in macrophages and granulocytes accounts, at least partly, for the neuroprotective effects observed in LysMCre-C/EBP $\beta^{\mathrm{fl} / \mathrm{fl}}$ mice in EAE. Unfortunately, specific inhibition of $C / E B P \beta$ in microglia is not feasible at present. Strategies aimed at targeting microglia, be it nanoparticles, vectors or other approaches, cannot avoid targeting also macrophages and often other myeloid cells [38]. Although we would favor a microglial-specific strategy, these results suggest that, if unavoidable, C/EBP $\beta$ inhibition not only in microglia but also in microglia-related cells would not be necessarily undesirable.

In contrast to mice with full $\mathrm{C} / \mathrm{EBP} \beta$ deficiency, LysMCre-C/EBP $\beta^{\mathrm{fl} / \mathrm{fl}}$ mice show normal fertility and survival. This is important in this study because it allowed us to obtain genetically homogenous litters of LysMCre-C/EBP $\beta^{\mathrm{fl} / \mathrm{fl}}$ pups from which to prepare primary microglial cultures in sufficient amounts to thoroughly characterize the role of $\mathrm{C} / \mathrm{EBP} \beta$ in microglial activation in vitro. In agreement with data from microglial cultures of $\mathrm{C} /$ EBP $\beta$-deficient mice [3], LysMCre-C/EBP $\beta^{\mathrm{f} / / \mathrm{fl}}$ microglia in culture showed normal growth and proliferation and reduced NOS2 expression and NO production upon LPS + IFN $\gamma$ challenge. Since NO production is an important contributor to AICD in microglia [25], we hypothesized that AICD, a mechanism of elimination of overactivated cells best described in T cells [39], could be attenuated in LysMCre-C/EBP $\beta^{\mathrm{f} / \mathrm{fl}}$ microglia. Strong AICD was observed in LPS + IFN $\gamma$-treated C/EBP $\beta^{\mathrm{f} / / \mathrm{l}}$ microglial cells, but this was unaffected by $C / E B P \beta$ absence indicating that $C / E B P \beta$ independent factors play important roles in microglial AICD. Since AICD is a protective mechanism that prevents overactivation [39], its maintenance in the absence of C/EBP $\beta$ could be a positive outcome of an eventual pharmacological strategy based on the inhibition of microglial C/EBP $\beta$.

The RNAseq analysis is the greatest leap forward this study provides on the characterization of the role of $\mathrm{C} /$ EBP $\beta$ in microglia. Various studies have analyzed the role of $C / E B P \beta$ on gene expression by microarrays after genetic deletion, inhibition by RNAi or overexpression in a variety of cell types. Particularly relevant are the studies that have shown important effects of $C / E B P \beta$ on LPS + IFN $\gamma^{-}$[40] or IFN $\gamma^{-}$[41] induced gene expression in macrophages. Studies in other cell types such as anaplastic large cell lymphoma have demonstrated 
a role or $\mathrm{C} / \mathrm{EBP} \beta$ in the expression of immune response genes [42]. The present study is the first to analyze the role of $C / E B P \beta$ in microglia with a transcriptomic approach and also the first one to analyze the role of $\mathrm{C} / \mathrm{EBP} \beta$ by RNAseq in any cell type. It shows massive changes in gene expression in microglia caused by the absence of $\mathrm{C} /$ EBP $\beta$. One thousand sixty-eight genes show significant differences in expression in C/EBP $\beta$-deficient microglia. Most of these genes, 867, were also affected by LPS \pm IFN $\gamma$ confirming the role of C/EBP $\beta$ in microglial activation, but interestingly, C/EBP $\beta$ absence affected the expression of 201 genes that were not affected by treatment, indicating a role for $C / E B P \beta$ also in the biology of nonactivated microglia. Gene Ontology enrichment analysis of the 1068 genes affected by microglial C/EBP $\beta$ deficiency identified five $\mathrm{GO}$ terms related to immune/inflammatory response among the 25 most significant $\mathrm{GO}$ terms. This is a strong confirmation of a key role for C/EBP $\beta$ in the regulation of the inflammatory gene program in microglia that others and we have proposed on the basis of the analysis of a limited number of genes [3, 7, 8, 24]. Relevant C/ EBP $\beta$-dependent genes among these classes include tolllike receptors and related proteins (Tlr1, Tlr7, Tlr8, Tlr9, Cd14, Cd180, Ly86, Nod2), cytokines (Il1a, Il12a, Il23a, Mif, Cxcl2, Cxcl3), cytokine receptors (Tnfrsf1a, Tnfrsf1b, Ccr1, Ccrl2, Csf1r) and enzymes such as inflammationrelated kinases (Rps6ka4, Mapkapk2), prostaglandin synthetic enzymes (Ptges, Ptgs2 $(=\mathrm{Cox} 2)$ ) or NADPH oxidase subunits (Cybb, Ncf1). A remarkable effect of $C / E B P \beta$ deficiency was observed in the expression of Nlrp3, a key component of the inflammasome [43]. Altogether, these findings show that the phenotype of LPS \pm IFN $\gamma$-treated microglia is markedly altered by the absence of $\mathrm{C} / \mathrm{EBP} \beta$, showing reduced responsiveness and attenuated responses in most arms of the pro-inflammatory program. KEGG pathway enrichment analysis also identified pathways related to inflammatory responses, such as chemokine signaling or cytokine-cytokine receptor interaction, but somewhat unexpectedly, three pathways related to phagocytosis among the five most significant KEGG pathways. Particularly interesting is the group of lysosomerelated genes. The significant genes in this group had a remarkably homogenous pattern of LPS \pm IFN $\gamma$-induced downregulation in $\mathrm{C} / \mathrm{EBP} \beta^{\mathrm{A} / \mathrm{fl}}$ microglia that was blunted in LysMCre-C/EBP $\beta^{\mathrm{fl} / \mathrm{fl}}$ microglia. These genes included degradative enzymes such as proteases (Ctss, Lgmn), nucleases (DNAse2a), sulfatases (Ids, Gns, Arsb) and glycosylases (Naga) and genes needed for lysosome acidification and assembly (Atp6v0a1, Atp6v0b, Ap1g2, Ap1s1, Ap3m2). The higher expression of lysosome genes may explain the improved ability of $\mathrm{C} / \mathrm{EBP} \beta$-deficient microglia to eliminate phagocytosed bacteria that we have observed. This pattern of attenuated pro-inflammatory gene expression and improved phagocytic and digesting capacity could be of interest in a neurodegeneration context where phagocytosis of cell debris and abnormal protein deposits is necessary, whereas chronic production of pro-inflammatory mediators can be detrimental.

The possibility to acutely isolate microglial cells from the adult CNS has allowed us to estimate the efficiency of $\mathrm{C} / \mathrm{EBP} \beta$ deletion in LysMCre-C/EBP $\beta^{\mathrm{fl} / \mathrm{fl}}$ microglia in vivo, and the data obtained shows it to be very high, close to $90 \%$. This is a critical point because it opens the possibility to use these mice to study for the first time the functional role of $\mathrm{C} / \mathrm{EBP} \beta$ in microglia in vivo which was the initial goal when generating this colony. In order to analyze the role of $\mathrm{C} / \mathrm{EBP} \beta$ on transcription of microglial genes in vivo, we selected a group of five pro-inflammatory genes (Tnfa, Il23a, Csf3, Ptges and Cybb) that (1) are regulated by $\mathrm{C} / \mathrm{EBP} \beta$ in microglia in culture as shown by RNAseq data; (2) have a well-established role in microglial activation; and (3) play a pathogenic role in EAE. Thus, genetic deletion of Ptges [44] and pharmacological inhibition of Tnfa [45] or Cybb [46] result in a significant attenuation of clinical symptoms of EAE, whereas genetic deletion of Il23a [47] or Csf3r [48] completely prevent the appearance of clinical EAE. The expression of these five genes in acutely isolated microglia was induced by systemic LPS injection in accordance with their pro-inflammatory character. Intriguingly, this expression was markedly attenuated in microglia isolated from LysMCre-C/EBP $\beta^{\mathrm{fl} / \mathrm{fl}}$ mice. This demonstrates that also in vivo $C / E B P \beta$ plays a major role in the regulation of pro-inflammatory gene expression in microglia.

Finally, robust neuroprotection from EAE was observed in LysMCre-C/EBP $\beta^{\mathrm{f} / \mathrm{f}}$ mice in three independent experiments. This neuroprotective effect is probably caused by the absence of C/EBP $\beta$ not only in microglia but also in monocyte-derived macrophages since both cell types are important in EAE pathogenesis $[49,50]$ and LysMCre will recombine in both [28]. The abovementioned proinflammatory genes Tnfa, Il23a, Csf3, Ptges and Cybb are candidates to mediate this effect, but given the role of $\mathrm{C} / \mathrm{EBP} \beta$ as transcription factor, we favor the idea that this effect is mediated by a higher number of genes directly or indirectly regulated by $\mathrm{C} / \mathrm{EBP} \beta$ and that $\mathrm{C} / \mathrm{EBP} \beta$ absence results in a global alteration of the phenotype of activated microglia/macrophages, as demonstrated by the RNAseq analysis in cultured microglia, rather than by an effect of one or a few genes. These data strongly support the search for strategies to selectively inhibit $\mathrm{C} / \mathrm{EBP} \beta$ in microglia or microglia/macrophages as potential therapies in neurological disorders with a strong neuroinflammatory component. The neuroprotection observed in LysMCre-C/ $E B P \beta^{\mathrm{fl} / \mathrm{fl}}$ mice in EAE together with the increased $\mathrm{C} /$ EBP $\beta$ expression in human multiple sclerosis samples point to this disease as a particularly suitable indication for such therapies. 


\section{Conclusion}

LysMCre-C/EBP $\beta \mathrm{fl} / \mathrm{fl}$ mice show robust C/EBP $\beta$ deletion in myeloid cells and are a good tool for the study of the role of $C / E B P \beta$ in these cells. In vivo and in vitro data support an important role for $\mathrm{C} / \mathrm{EBP} \beta$ in the regulation of pro-inflammatory responses in microglia. Moreover, RNAseq results demonstrate the implication of C/EBP $\beta$ also in other cellular programs in activated microglia and also in non-activated microglia. Finally, C/EBP $\beta$ absence results in attenuated EAE supporting a therapeutic potential for $\mathrm{C} / \mathrm{EBP} \beta$ inhibition.

\section{Additional files}

Additional file 1: Tables S1-S6. List the genes significantly up-regulated (tables 1, 3 and 5) or down-regulated (tables 2,4 and 6) by the absence of C/EBP $\beta$ in control (tables 1, 2), LPS-treated (tables 3,4) and LPS+IFNy-treated (tables 5,6 ) primary microglial cultures. These data were obtained by RNAseq as described in Methods. (ZIP $253 \mathrm{~kb}$ )

Additional file 2: Figure S1. Weighted Gene Correlation Network Analyses of microglial activation, treatment and LysMCre-C/EBP $\beta^{\mathrm{fl} / f \mathrm{l}}$ phenotype. A) Correlation dendrogram of genes. WGCNA algorithm was applied to filtered expression of all samples, a soft-threshold for the similarity matrix of $\beta=9$ was used, and module detection was obtained with a dynamic tree cut; color bar down of the dendrogram shows module pertainance of genes with a large turquoise module corresponding to genes downregulated upon treatment with either LPS or LPS + IFNץ. B) Hierarchical clustering of detected modules by WGCNA and genotype and treatment traits; module MEyellow is the closest group of genes related to the treatment trait, whereas MEviolet is for genotype. C) Module violet heatmap (top) and eigengene expression graph (bottom) module violet is the closest group of genes related to genotype effect, contains C/EBP $\beta$ and Lyz2. D) Metacore ${ }^{\mathrm{TM}}$ network obtained by literature described interactions among the genes in module violet evidences a network of genes described to interact with $C / E B P \beta$ as a central hub. E) Correlation network obtained with a threshold for distance of 0.3 , with C/ $\mathrm{EBP} \beta$ as a central hub (node size relative to degree of node). (TIF $1462 \mathrm{~kb}$ )

\section{Abbreviations \\ AICD: Activation-induced cell death; C/EBPB: CCAAT/enhancer-binding protein $\beta$; DEG: Differentially expressed genes; EAE: Experimental autoimmune encephalomyelitis; GO: Gene Ontology; IFNy: Interferon Y; KEGG: Kyoto Encyclopedia of Genes and Genomes; LPS: Lipopolysaccharide; LysM: Lysozyme M; MOG: Myelin oligodendrocyte glycoprotein peptide; PCR: Polymerase chain reaction; qRT-PCR: Quantitative real-time PCR; RNAseq: RNA sequencing; SEM: Standard error of the mean; WGCNA: Weighted Correlation Gene Network Analysis}

\section{Acknowledgements}

The authors thank Esta Sterneck (National Cancer Institute, USA) for providing the $C / E B P \beta^{f / f l l}$ mice, the staff of the Animal Facilities of the School of Medicine (University of Barcelona) for the professional care of the mouse colonies, Ellen Gelpí (Banc de Teixits Neurològics, Hospital Clinic-Universitat de Barcelona, IDIBAPS) for providing the human samples, Pep Tusell (IIBB, CSIC) for the technical assistance, Maria Iñíguez and Alberto de Luis (Genomic Plataform, CIBIR) for the sequencing of RNA samples, John $\mathrm{H}$. Brumell (Hospital for Sick Children, Toronto, Canada) for the pBR.RFP.1 plasmid and Antonio Juárez (University of Barcelona) for the Salmonella typhimurium strain.

\section{Funding}

MPS was a recipient of a FPU grant AP2010-5428 from the Spanish Ministerio de Educacion, Cultura y Deporte. This study was supported by grants PI07/ 455, PI10/378, PI12/709 and PI14/302 from the Instituto de Salud Carlos III, Spain, cofinanced with FEDER funds, and a grant from La Marató-TV3.

\section{Availability of data and materials}

RNAseq datasets were deposited in the Gene Expression Omnibus database under dataset accession number GSE90046. Other data supporting the conclusions of this study will be available upon reasonable request.

\section{Authors' contributions}

MPS performed most of the experimental work and data analysis. JMVT and GGDB did most of the RNAseq analysis. JSe participated in the ex vivo isolation of the microglia and cytospin. TV participated in the EAE experiments and did most of the work on the human samples. PC did most of the histology work. JM and AFV participated in the phagocytosis experiments. MS initiated the generation of the LysMCre-C/EBP $\beta^{\mathrm{fl} / \mathrm{fl}}$ colony. JMC participated in the RNAseq analysis. CS participated in the EAE experiments. JSa conceived the project, participated in the EAE experiments and wrote the most of the manuscript. MPS, JMVT, TV, MS, JSe, CS and JSa provided input and ideas throughout the process. MPS and GGDB wrote parts of the manuscript. All authors revised and approved the final manuscript.

\section{Competing interests}

The authors declare that they have no competing interests.

\section{Consent for publication}

Not applicable.

\section{Ethics approval}

Postmortem human temporal cortex samples were supplied by the Neurological Tissue Bank of the Biobanc-Hospital Clínic-IDIBAPS in accordance with the Helsinki Declaration, Convention of the Council of Europe on Human Rights and Biomedicine and Ethical Committee of the University of Barcelona. All procedures involving animals were approved by the Ethical Committee for Animal Experimentation of the Universitat de Barcelona and by the Commission for Animal Experimentation of the Generalitat de Catalunya, with protocol numbers DAAM 5026, 7064 and 7065.

\section{Publisher's Note}

Springer Nature remains neutral with regard to jurisdictional claims in published maps and institutional affiliations.

\section{Author details}

${ }^{1}$ Department of Biomedicine, Biochemistry and Molecular Biology Unit, School of Medicine, University of Barcelona, IDIBAPS, Barcelona, Spain. ${ }^{2}$ Department of Biomedicine, Histology Unit, School of Medicine, University of Barcelona, IDIBAPS, Barcelona, Spain. ${ }^{3}$ Department of Cerebral Ischemia and Neurodegeneration, Institut d'Investigacions Biomèdiques de Barcelona, CSIC, IDIBAPS, Barcelona, Spain. ${ }^{4}$ Department of Pathology, Hospital Clinic, ISGlobal, CRESIB, Barcelona, Spain. ${ }^{5}$ Department of Physiology and Immunology, School of Biology, University of Barcelona, Barcelona, Catalonia, Spain. ${ }^{6}$ Institute of Neurosciences, University of Barcelona, Barcelona, Spain.

Received: 11 November 2016 Accepted: 6 March 2017

Published online: 16 March 2017

References

1. Block ML, Zecca L, Hong JS. Microglia-mediated neurotoxicity: uncovering the molecular mechanisms. Nat Rev Neurosci. 2007:8:57-69.

2. Cartier N, Lewis CA, Zhang R, Rossi FM. The role of microglia in human disease: therapeutic tool or target? Acta Neuropathol. 2014;128:363-80.

3. Straccia M, Gresa-Arribas N, Dentesano G, Ejarque-Ortiz A, Tusell JM, Serratosa J, Sola C, Saura J. Pro-inflammatory gene expression and neurotoxic effects of activated microglia are attenuated by absence of CCAAT/enhancer binding protein beta. J Neuroinflammation. 2011;8:156.

4. Pulido-Salgado M, Vidal-Taboada JM, Saura J. C/EBPbeta and C/EBPdelta transcription factors: basic biology and roles in the CNS. Prog Neurobiol. 2015.

5. Ejarque-Ortiz A, Medina MG, Tusell JM, Perez-Gonzalez AP, Serratosa J, Saura J. Upregulation of CCAAT/enhancer binding protein beta in activated astrocytes and microglia. Glia. 2007;55:178-88.

6. Dasgupta S, Jana M, Liu X, Pahan K. Role of very-late antigen-4 (VLA-4) in myelin basic protein-primed T cell contact-induced expression of proinflammatory cytokines in microglial cells. J Biol Chem. 2003;278:22424-31.

7. Cortes-Canteli M, Luna-Medina R, Sanz-Sancristobal M, Alvarez-Barrientos A, Santos A, Perez-Castillo A. CCAAT/enhancer binding protein beta deficiency 
provides cerebral protection following excitotoxic injury. J Cell Sci. 2008;121: 1224-34.

8. Straccia M, Dentesano G, Valente T, Pulido-Salgado M, Sola C, Saura J. CCAAT/enhancer binding protein beta regulates prostaglandin $\mathrm{E}$ synthase expression and prostaglandin E2 production in activated microglial cells. Glia. 2013;61:1607-19.

9. Kapadia R, Tureyen K, Bowen KK, Kalluri H, Johnson PF, Vemuganti R. Decreased brain damage and curtailed inflammation in transcription facto CCAAT/enhancer binding protein beta knockout mice following transient focal cerebral ischemia. J Neurochem. 2006;98:1718-31.

10. Ericsson C, Nister M. Protein extraction from solid tissue. Methods Mol Biol. 2011;675:307-12.

11. Sterneck E, Zhu S, Ramirez A, Jorcano JL, Smart RC. Conditional ablation of C/EBP beta demonstrates its keratinocyte-specific requirement for cell survival and mouse skin tumorigenesis. Oncogene. 2006;25:1272-6.

12. Saura J, Tusell JM, Serratosa J. High-yield isolation of murine microglia by mild trypsinization. Glia. 2003;44:183-9.

13. Nikodemova M, Watters JJ. Efficient isolation of live microglia with preserved phenotypes from adult mouse brain. J Neuroinflammation. 2012;9:147.

14. Banos RC, Vivero A, Aznar S, Garcia J, Pons M, Madrid C, Juarez A. Differential regulation of horizontally acquired and core genome genes by the bacterial modulator H-NS. PLoS Genet. 2009;5, e1000513.

15. Birmingham $\mathrm{CL}$, Smith AC, Bakowski MA, Yoshimori T, Brumell JH. Autophagy controls Salmonella infection in response to damage to the Salmonella-containing vacuole. J Biol Chem. 2006;281:11374-83.

16. Li H, Handsaker B, Wysoker A, Fennell T, Ruan J, Homer N, Marth G, Abecasis G, Durbin R. The Sequence Alignment/Map format and SAMtools. Bioinformatics. 2009;25:2078-9.

17. Liao Y, Smyth GK, Shi W. The Subread aligner: fast, accurate and scalable read mapping by seed-and-vote. Nucleic Acids Res. 2013;41, e108.

18. Liao Y, Smyth GK, Shi W. featureCounts: an efficient general purpose program for assigning sequence reads to genomic features. Bioinformatics. 2014;30:923-30.

19. Law CW, Chen Y, Shi W, Smyth GK. voom: precision weights unlock linear model analysis tools for RNA-seq read counts. Genome Biol. 2014;15:R29.

20. Ritchie ME, Phipson B, Wu D, Hu Y, Law CW, Shi W, Smyth GK. limma powers differential expression analyses for RNA-sequencing and microarray studies. Nucleic Acids Res. 2015;43, e47.

21. Carmona-Saez P, Chagoyen M, Tirado F, Carazo JM, Pascual-Montano A. GENECODIS: a web-based tool for finding significant concurrent annotations in gene lists. Genome Biol. 2007:8:R3.

22. Langfelder P, Horvath S. WGCNA: an R package for weighted correlation network analysis. BMC Bioinformatics. 2008;9:559.

23. Screpanti I, Romani L, Musiani P, Modesti A, Fattori E, Lazzaro D, Sellitto C, Scarpa S, Bellavia D, Lattanzio G, et al. Lymphoproliferative disorder and imbalanced T-helper response in C/EBP beta-deficient mice. EMBO J. 1995 14:1932-41.

24. Jana M, Liu X, Koka S, Ghosh S, Petro TM, Pahan K. Ligation of CD40 stimulates the induction of nitric-oxide synthase in microglial cells. J Biol Chem. 2001;276:44527-33.

25. Mayo L, Stein R. Characterization of LPS and interferon-gamma triggered activation-induced cell death in N9 and primary microglial cells: induction of the mitochondrial gateway by nitric oxide. Cell Death Differ. 2007;14:183-6.

26. Valente T, Mancera P, Tusell JM, Serratosa J, Saura J. C/EBP $\beta$ expression in activated microglia in amyotrophic lateral sclerosis. Neurobiol Aging. 2012; 33:2186-99.

27. Clausen BE, Burkhardt C, Reith W, Renkawitz R, Forster I. Conditional gene targeting in macrophages and granulocytes using LysMcre mice. Transgenic Res. 1999:8:265-77

28. Wieghofer P, Knobeloch KP, Prinz M. Genetic targeting of microglia. Glia. 2015;63:1-22.

29. Goldmann T, Wieghofer P, Muller PF, Wolf Y, Varol D, Yona S, Brendecke SM, Kierdorf K, Staszewski O, Datta M, et al. A new type of microglia gene targeting shows TAK1 to be pivotal in CNS autoimmune inflammation. Nat Neurosci. 2013;16:1618-26.

30. Hucke S, Flossdorf J, Grutzke B, Dunay IR, Frenzel K, Jungverdorben J, Linnartz $B$, Mack M, Peitz M, Brustle O, et al. Licensing of myeloid cells promotes central nervous system autoimmunity and is controlled by peroxisome proliferatoractivated receptor gamma. Brain. 2012;135:1586-605.

31. Willemen HL, Eijkelkamp N, Wang H, Dantzer R, Dorn 2nd GW, Kelley KW, Heijnen CJ, Kavelaars A. Microglial/macrophage GRK2 determines duration of peripheral IL-1 beta-induced hyperalgesia: contribution of spinal cord CX3CR1, p38 and IL-1 signaling. Pain. 2010;150:550-60.

32. Qin H, Yeh WI, De Sarno P, Holdbrooks AT, Liu Y, Muldowney MT, Reynolds $\mathrm{SL}$, Yanagisawa LL, Fox 3rd TH, Park K, et al. Signal transducer and activator of transcription-3/suppressor of cytokine signaling-3 (STAT3/SOCS3) axis in myeloid cells regulates neuroinflammation. Proc Natl Acad Sci U S A. 2012; 109:5004-9.

33. Cho IH, Hong J, Suh EC, Kim JH, Lee H, Lee JE, Lee S, Kim CH, Kim DW, Jo EK, et al. Role of microglial IKKbeta in kainic acid-induced hippocampal neuronal cell death. Brain. 2008;131:3019-33.

34. Derecki NC, Cronk JC, Lu Z, Xu E, Abbott SB, Guyenet PG, Kipnis J. Wild-type microglia arrest pathology in a mouse model of Rett syndrome. Nature. 2012:484:105-9.

35. Kaindl AM, Degos V, Peineau S, Gouadon E, Chhor V, Loron G, Le Charpentier T, Josserand J, Ali C, Vivien D, et al. Activation of microglial $\mathrm{N}$-methyl-D-aspartate receptors triggers inflammation and neuronal cell death in the developing and mature brain. Ann Neurol. 2012;72:536-49.

36. Prinz M, Schmidt H, Mildner A, Knobeloch KP, Hanisch UK, Raasch J, Merkler D, Detje C, Gutcher I, Mages J, et al. Distinct and nonredundant in vivo functions of IFNAR on myeloid cells limit autoimmunity in the central nervous system. Immunity. 2008;28:675-86.

37. Huber R, Pietsch D, Panterodt T, Brand K. Regulation of C/EBPbeta and resulting functions in cells of the monocytic lineage. Cell Signal. 2012;24:1287-96.

38. Biber K, Moller T, Boddeke E, Prinz M. Central nervous system myeloid cells as drug targets: current status and translational challenges. Nat Rev Drug Discov. 2015.

39. Arakaki R, Yamada A, Kudo Y, Hayashi Y, Ishimaru N. Mechanism of activation-induced cell death of $T$ cells and regulation of FasL expression. Crit Rev Immunol. 2014;34:301-14.

40. Akagi T, Thoennissen NH, George A, Crooks G, Song JH, Okamoto R, Nowak D, Gombart AF, Koeffler HP. In vivo deficiency of both C/EBPbeta and C/ EBPepsilon results in highly defective myeloid differentiation and lack of cytokine response. PLoS One. 2010;5, e15419.

41. Gade P, Roy SK, Li H, Nallar SC, Kalvakolanu DV. Critical role for transcription factor C/EBP-beta in regulating the expression of death-associated protein kinase 1. Mol Cell Biol. 2008;28:2528-48.

42. Bonzheim I, Irmler M, Klier-Richter M, Steinhilber J, Anastasov N, Schafer S, Adam P, Beckers J, Raffeld M, Fend F, Quintanilla-Martinez L. Identification of C/EBPbeta target genes in ALK+ anaplastic large cell lymphoma (ALCL) by gene expression profiling and chromatin immunoprecipitation. PLoS One. 2013;8, e64544.

43. Jo EK, Kim JK, Shin DM, Sasakawa C. Molecular mechanisms regulating NLRP3 inflammasome activation. Cell Mol Immunol. 2015.

44. Kihara Y, Matsushita T, Kita Y, Uematsu S, Akira S, Kira J, Ishii S, Shimizu T. Targeted lipidomics reveals mPGES-1-PGE2 as a therapeutic target for multiple sclerosis. Proc Natl Acad Sci U S A. 2009;106:21807-12.

45. Brambilla R, Ashbaugh JJ, Magliozzi R, Dellarole A, Karmally S, Szymkowski DE, Bethea JR. Inhibition of soluble tumour necrosis factor is therapeutic in experimental autoimmune encephalomyelitis and promotes axon preservation and remyelination. Brain. 2011;134:2736-54.

46. Chechneva OV, Mayrhofer F, Daugherty DJ, Pleasure DE, Hong JS, Deng W. Low dose dextromethorphan attenuates moderate experimental autoimmune encephalomyelitis by inhibiting NOX2 and reducing peripheral immune cells infiltration in the spinal cord. Neurobiol Dis. 2011;44:63-72.

47. Thakker P, Leach MW, Kuang W, Benoit SE, Leonard JP, Marusic S. IL-23 is critical in the induction but not in the effector phase of experimental autoimmune encephalomyelitis. J Immunol. 2007:178:2589-98.

48. Rumble JM, Huber AK, Krishnamoorthy G, Srinivasan A, Giles DA, Zhang X, Wang L, Segal BM. Neutrophil-related factors as biomarkers in EAE and MS. J Exp Med. 2015;212:23-35.

49. Schwartz M, Deczkowska A. Neurological disease as a failure of brainimmune crosstalk: the multiple faces of neuroinflammation. Trends Immunol. 2016:37:668-79.

50. Constantinescu CS, Farooqi N, O'Brien K, Gran B. Experimental autoimmune encephalomyelitis (EAE) as a model for multiple sclerosis (MS). Br J Pharmacol. 2011:164:1079-106. 